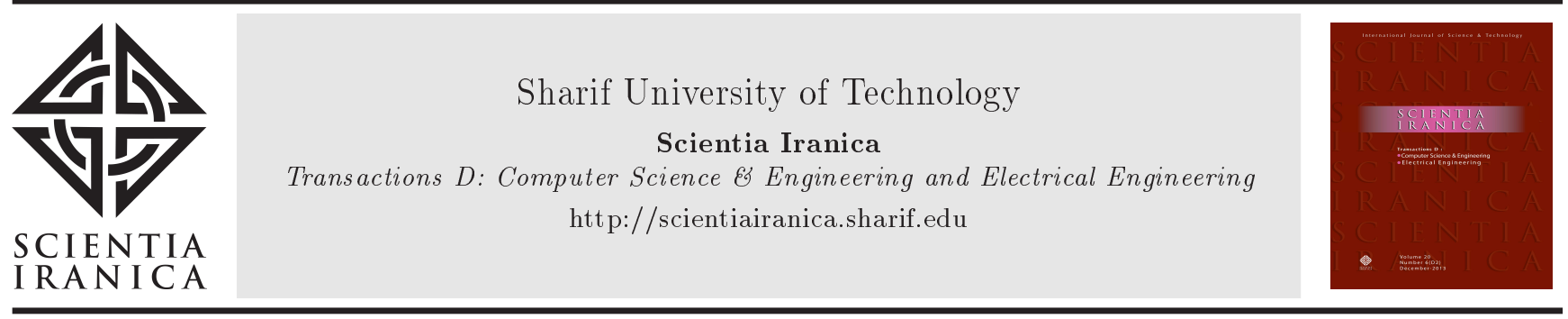

\title{
A modified variant of grey wolf optimizer
}

\author{
N. Singh \\ Department of Mathematics, Punjabi University, Patiala-147002, Punjab, India. \\ Received 27 December 2017; received in revised form 5 April 2018; accepted 2 July 2018
}

\author{
KEYWORDS \\ Particle Swarm \\ Optimization (PSO); \\ Grey Wolf \\ Optimization (GWO); \\ Mean grey wolf \\ optimization; \\ Meta-heuristics.
}

\begin{abstract}
The original version of Grey Wolf Optimization (GWO) algorithm has a few disadvantages such as low solving accuracy, unsatisfactory ability of local searching, and slow convergence rate. In order to compensate these disadvantages of grey wolf optimizer, a new version of grey wolf optimizer algorithm was proposed by modifying the encircling behavior and position update equations of GWO algorithm. The accuracy and convergence performances of the modified variant were tested on several well-known classical, sine datasets, and cantilever beam design functions. For verification, the results were compared with some of the most powerful, well-known algorithms, i.e., particle swarm optimization, grey wolf optimizer, and mean grey wolf optimization. The experimental solutions demonstrated that the modified variant was able to provide very comparable solutions in terms of improved minimum value of objective function, maximum value of objective function, mean, standard deviation, and convergence rate.

(C) 2020 Sharif University of Technology. All rights reserved.
\end{abstract}

\section{Introduction}

Over the last few decades, population-inspired metaheuristics have received much attention. Several nature-inspired meta-heuristics have been proposed, such as Genetic Algorithm (GA) [1], Particle Swarm Optimization (PSO) [2], and Differential Evolution (DE) [3,4]. Although these meta-heuristics are competent enough to find the solution to complex optimization functions, there is no optimization technique for finding the solutions to all types of functions based on the no free lunch theorem [5]. Therefore, the theorem allows scientists to develop several new nature-inspired techniques. Various recent meta-heuristics include Artificial Bee Colony (ABC) algorithm [6], Cuckoo Search (CS) algorithm [7], Gravitational Search Algorithm (GSA) [8], firefly algorithm [9], cuckoo optimization algorithm [10], adaptive Gbest-guided Gravitational

*. Corresponding author.

E-mail address: narindersinghgoria@ymail.com (N. Singh).

doi: $10.24200 /$ sci.2018.50122.1523
Search Algorithm (GGSA) [11], Grey Wolf Optimization (GWO) [12], Ant Lion Optimizer (ALO) [13], Multiverse Optimizer (MVO) [14], Shuffled Frog-Leaping Algorithm (SFLA) [15], Bacterial Foraging Optimization Algorithm (BFOA) [16], Opposition-based Grey Wolf Optimization (OGWO) [17], one half personal best position particle swarm optimizations [18], half mean particle swarm optimization algorithm [19], personal best position particle swarm optimization [20], Hybrid Particle Swarm Optimization (HPSO) [21], hybrid Mean Gbest Particle Swarm Optimization Gravitational Search Algorithm (MGBPSO-GSA) [22], Mean Grey Wolf Optimization (MGWO) [23], Hybrid Particle Swarm Optimization Grey Wolf Optimization (HPSOGWO) [24], Hybrid Grey Wolf Optimization Sine Cosine Algorithm (HGWOSCA) [25], Hybrid Algorithm Grey Wolf Optimization (HAGWO) [26], and many others.

The biogeography-based optimization algorithm proposed by Simon [27] is a new population-based variant, which studies the geographical distribution of biological organisms. The biogeography-based optimization approach adopts a migration operator to 
share information between solutions. This aspect is the same as that of other nature-inspired variants, i.e., GA and PSO. The performance of the BiogeographyBased Optimization (BBO) variant has been compared with 14 benchmark functions and a real-life sensor selection problem. On the basis of obtaining statistical results, it has been observed that the existing variant produced better quality of solutions that outperformed other recent meta-heuristics.

Bat Algorithm (BA) was proposed by Yang [28]. $\mathrm{BA}$ is a bio-inspired variant and has been found very efficient. This variant mimics the echolocation ability of microbat that uses it to navigate and hunt. The position of the bat provides a probable solution to the problem. The fitness of the solution is specified by the best position of a bat to its prey. BA has many advantages over other variants including a number of tunable parameters that provide greater control over the optimization process.

Flower Pollination Algorithm (FPA) was first proposed by Yang [29]. FPA is inspired by the pollination process of flowers. The performance of this variant was tested on ten test functions, and results were compared with those obtained using PSO and GA. On the basis of the simulation results, one can observe that the flower algorithm is more efficient than both PSO and GA are. Furthermore, authors use this variant to solve a nonlinear problem, showing that the convergence rate is almost exponential.

Recently, Mirjalili [30] proposed the GWO algorithm for eight dataset functions, and its performance was compared with other nature-inspired algorithms. On the basis of statistical results, it was proved that the GWO variant provided highly competitive results in terms of improved local optima avoidance.

MGWO was proposed by Singh and Singh [31]. This variant was developed by modifying the position update (encircling behavior) equations of GWO algorithm. MGWO variant was tested on several well-known tests (unimodal, multimodal, and fixeddimension multimodal functions); moreover, the performance of the modified variant was compared with those of PSO and GWO. In addition, five datasets were classified to assess the accuracy of the modified variant. The obtained results were compared with those obtained by many different meta-heuristic approaches, i.e., GWO, PSO, Population-Based Incremental Learning (PBIL), Ant Colony Optimization (ACO), etc. According to the statistical results, it was observed that the modified variant could find the best solutions in terms of high accuracy level in classification and improved local optima avoidance.

Mittal et al. [32] developed a modified variant of the GWO, called Modified GWO, which focused on proper balance amid exploitation and exploration that led to the optimum accuracy of the variant. The simu- lations based on standard functions and real-life application demonstrated the verified efficiency and stability of the existing variant based on the basic grey wolf optimizer algorithm and some recent meta-heuristics.

GWO is a newly developed population-based approach inspired by the leadership hierarchy and hunting mechanism of grey wolves in nature and has been effectively applied to solve feature subset selection [33], economic dispatch problems [34], flow shop scheduling problem [35], optimal design of double-layer grids [36], time forecasting [37], optimizing key values in the cryptography algorithms [38], and optimal power flow problem [39]. A number of the nature-inspired algorithms are also developed to improve the performance of basic GWO that include a hybrid version of GWO with PSO [40], binary GWO [41], parallelized GWO $[42,43]$, and integration of DE with GWO [44].

$\mathrm{Li}$ et al. [45] proposed a modified discrete GWO variant to realize the multi-level image segmentation and optimize image histograms. Based on the high efficiency of grey wolf optimizer in the course of stability and optimization, this article effectively applied the Modified Discrete Grey Wolf Optimizer (MDGWO) algorithm to the field of Machine Translation (MT) by improving the location of the agents during the hunting and using weights to optimize the final position of prey. The MDGWO approach not only obtains better segmentation quality, but also proves its obvious superiority over $\mathrm{ABC}, \mathrm{DE}, \mathrm{GWO}$, and Multilevel Thresholding Electromagnetism-like Optimization (MTEMO) in terms of accuracy, multilevel thresholding, and stability.

Liu et al. [46] developed an intelligent grey wolf optimizer variant, called DCS-GWO, by combining qthresholding with the GWO variant. In this variant, positions of the grey wolves were initialized by the qthresholding approach and updated by using the idea of GWO. The experimental solutions illustrated that the existing variant enjoyed better recovery accuracy than previous greedy pursuit approaches at the expense of computational complexity.

Mirjalili et al. [47] proposed two novel optimization techniques, Salp Swarm Algorithm (SSA) and Multi-objective Salp Swarm Algorithm (MSSA), for finding the solution of global optimization functions with multiple and single objectives. The main inspiration of SSA and MSSA is the swarming behavior of Salps when navigating and foraging in the ocean. The performance of the existing variant was tested on several standard and real-life applications. Based on the solutions of the existing variant, it was proven that this variant could obtain approximately Pareto optimal results with high convergence and coverage.

Raj and Bhattacharyya [48] applied several recent meta-heuristics to achieve the best possible optimal solution for reactive power planning with FACTS 
devices. Further, some more recent techniques have been also applied to find the best optimal setting of all control variables. The working performance of the existing variant was illustrated by comparing the solutions obtained with all other recent meta-heuristics. Based on the simulation results, the existing variant showed few generations, which do not get trapped in the local minima, and offered promising convergence characteristics.

This article focuses on grey wolf optimizer, developed by Mirjalili et al. [12] in 2014, based on the simulation of hunting behavior and social leadership of grey wolves in nature. Experimental results proved that the better accuracy of the existing variant was also comparable to that of other meta-heuristics. Since it is easy and simple to implement and has fewer control constants, grey wolf optimizer has received much attention and used to find the solution of practical real-life functions.

PSO, GA, evolutionary algorithm, differential algorithm, and ACO are the most popular meta-heuristic global optimization approaches. These nature-inspired techniques expand the search area dimension, while grey wolf optimizer provides an unsatisfactory convergence behavior regarding exploitation $[49,50]$. Hence, it is essential to emphasize that our research effort revolves around the increase of the local search ability of grey wolf optimizer technique. In order to improve the local search ability of the GWO algorithm, a newly modified meta-heuristic is proposed in this research, and its performance is compared with the performance results of grey wolf optimizer and some other recent nature-inspired algorithms; ultimately, Modified Variant of Grey Wolf Optimization (MVGWO) performs significantly better.

The rest of the paper is structured as follows. Section 2 describes the GWO algorithm. Section 3 presents the newly proposed algorithm, MVGWO. The MVGWO mathematical model and pseudocode are discussed in Section 3. The tested Unimodal, Multimodal, and Fixed-dim Multimodal classical functions are presented in Section 4. Results and discussion are summarized in Sections 5 and 6, respectively. Sine dataset and cantilever beam design functions are briefly described in Sections 7 and 8. Conclusions are drawn on the basis of the results obtained, as will be presented in Section 9.

\section{Grey wolf optimization algorithm}

The grey wolf optimizer algorithm is a new global optimization approach that simulates the grey wolves leadership and hunting in nature. These approaches have been inspired by simple concepts.

Mirjalili, et al. [12] proposed a GWO metaheuristic approach. The GWO variant mimics the hunting mechanism and leadership hierarchy of grey wolves in nature. In the hierarchy of GWO, alpha is considered as the dominating agent among the group. The rest of the subordinates to alpha include beta and delta that help control the majority of wolves in the hierarchy that are considered as omega.

In addition, three main steps of hunting, searching for prey, encircling prey, and attacking prey, are implemented to perform optimization.

The encircling behavior of each member of the population is represented by the following mathematical equations:

$$
\begin{aligned}
& d=\left|c \cdot x_{p(t)}-x(t)\right|, \\
& x(t+1)=x_{p(t)}-a . d,
\end{aligned}
$$

where $x_{p}$ is the position vector of the prey, $t$ is the time, and $x$ indicates the position vector of a grey wolf.

Vectors $a$ and $c$ are mathematically calculated as follows:

$$
\begin{aligned}
& a=2 l . r_{1}-l, \\
& c=2 . r_{2},
\end{aligned}
$$

where the above components are linearly decreased from 2 to 0 over the course of generations, and $r_{1} \cdot r_{2} \in$ $[0,1]$ are random vectors.

Hunting: In order to mathematically simulate the hunting behavior, it is supposed that alpha $(\alpha)$, beta $(\beta)$, and delta $(\delta)$ have better knowledge about the potential location of prey. The following mathematical equations are developed in this regard:

$$
\begin{aligned}
& \vec{d}_{\alpha}=\left|\vec{c}_{1} \cdot \vec{x}_{\alpha}-\vec{x}\right|, \quad \vec{d}_{\beta}=\left|\vec{c}_{2} \cdot \vec{x}_{\beta}-\vec{x}\right|, \\
& \vec{d}_{\delta}=\left|\vec{c}_{3} \cdot \vec{x}_{\delta}-\vec{x}\right|, \\
& \vec{x}_{1}=\vec{x}_{\alpha}-\vec{a}_{1} \cdot\left(\vec{d}_{\alpha}\right), \quad \vec{x}_{2}=\vec{x}_{\beta}-\vec{a}_{2} \cdot\left(\vec{d}_{\beta}\right), \\
& \vec{x}_{3}=\vec{x}_{\delta}-\vec{a}_{3} \cdot\left(\vec{d}_{\delta}\right), \\
& \frac{\vec{x}_{1}+\vec{x}_{2}+\vec{x}_{3}}{3}
\end{aligned}
$$

where $\vec{x}_{\alpha}, \vec{x}_{\beta}$, and $\vec{x}_{\delta}$ are the positions of the member of the population in the searching space at the $t$ th iteration, $t$ indicates the current iteration, and $\vec{x}(t)$ presents the position of the grey wolf at the $t$ th iteration:

$$
\begin{aligned}
& \vec{a}_{(.)}=2 \vec{l} \cdot \vec{r}_{1}-\vec{l}, \\
& \vec{c}_{(.)}=2 \cdot \vec{r}_{2},
\end{aligned}
$$

where components of $\vec{l}$ are linearly decreased from 2 to 0 over the course of generations, and $r_{1}, r_{2}$ are random vectors in $[0,1]$. In addition, $\vec{a}_{(.)}$and $\vec{c}_{(.)}$are the coefficient vectors of alpha $(\alpha)$, beta $(\beta)$, and delta $(\delta)$ wolfs. 
Searching for prey and attacking prey: $A$ is a random value in the gap $(-a, a)$. When random value $|A|<1$, the wolves are forced to attack the prey. Searching for prey is an exploration ability, and attacking the prey is an exploitation ability. Arbitrary values of are utilized to force the search to move away from the prey.

When $|A|>1$, the members of the population are enforced to diverge from the prey.

\section{Modified variant of grey wolf optimizer}

Mirjalili et al. [14] proposed a new version of population-based algorithms, called GWO. The GWO variant mimics the hunting mechanism and leadership hierarchy of grey wolves in nature. In the hierarchy of GWO, alpha is considered the dominating agent among the group. The rest of the subordinates to alpha include beta and delta that help control the majority of wolves in the hierarchy that are considered as omega. In addition, three main steps of hunting, i.e., searching for prey, encircling prey, and attacking prey, are implemented to perform optimization.

The proposed variant has been developed by modifying encircling behavior and position update equation of GWO algorithm with the aim of improving the performance, convergence speed, and accuracy of the grey wolf optimizer meta-heuristics. In the MVGWO, the population is divided into five different groups, such as alpha, beta, gamma, delta, and omega, which are employed for simulating the leadership hierarchy (see in Figure 1). The rest of all operations are the same as grey wolf optimizer variant [14].

Social Hierarchy: In order to develop the proposed mathematical model, the social hierarchy of wolves is considered when designing an MVGWO, where the fittest solution is alpha. Accordingly, the second, third, and fourth best solutions are named beta, gamma, and delta. The rest of the agent solutions are assumed to be omega.

The mathematical model of the encircling behav-

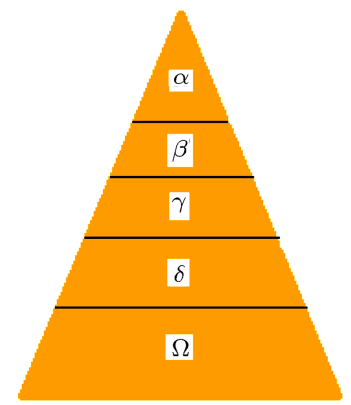

Figure 1. Hierarchy of grey wolf (dominance decreases from top to down). ior is represented by the following equations:

$$
\begin{aligned}
& d=\left|c \cdot x_{p(t)}-\mu \times x(t)\right|, \\
& x(t+1)=x_{p(t)}-a . d,
\end{aligned}
$$

where coefficient vectors, $a$ and $c$, are given by:

$$
\begin{aligned}
& a=2 l \cdot \vec{r}_{1}, \\
& c=2 \cdot \vec{r}_{2},
\end{aligned}
$$

where the components are as follows: $l \in[2,0]$ and $\vec{r}_{1} \cdot \vec{r}_{2} \in[0,1]$.

Hunting: In order to simulate the hunting behavior mathematically, it is supposed that alpha $(\alpha)$, beta $(\beta)$, gamma $(\gamma)$, and delta $(\delta)$ have better knowledge about the potential location of prey. The following mathematical equations are developed in this regard:

$$
\begin{aligned}
& \vec{d}_{\alpha}=\left|\vec{c}_{1} \cdot \vec{x}_{\alpha}-\vec{x}\right|, \quad \vec{d}_{\beta}=\left|\vec{c}_{2} \cdot \vec{x}_{\beta}-\vec{x}\right|, \\
& \vec{d}_{\gamma}=\left|\vec{c}_{3} \cdot \vec{x}_{\gamma}-\vec{x}\right|, \quad \vec{d}_{\delta}=\left|\vec{c}_{4} \cdot \vec{x}_{\delta}-\vec{x}\right|, \\
& \vec{x}_{1}=\vec{x}_{\alpha}-\vec{a}_{1} \cdot\left(\vec{d}_{\alpha}\right), \quad \vec{x}_{2}=\vec{x}_{\beta}-\vec{a}_{2} \cdot\left(\vec{d}_{\beta}\right), \\
& \vec{x}_{3}=\vec{x}_{\gamma}-\vec{a}_{3} \cdot\left(\overrightarrow{d_{\gamma}}\right), \quad \vec{x}_{4}=\vec{x}_{\delta}-\vec{a}_{4} \cdot\left(\vec{d}_{\delta}\right), \\
& \frac{\vec{x}_{1}+\vec{x}_{2}+\vec{x}_{3}+\vec{x}_{4}}{4}, \\
& \vec{a}_{(.)}=2 \vec{l} \cdot \vec{r}_{1}-\vec{l}, \\
& \vec{c}_{(.)}=2 \cdot \vec{r}_{2} .
\end{aligned}
$$

\section{Pseudo Code of MVGWO:}

Initialization of population

Initialize $l, a$, and $c$

Evaluate the fitness of each search member $x_{\alpha}, x_{\beta}, x_{\gamma}$, and $x_{\delta}$ as the first, second, third and fourth best search members while $(t<\max$ no. of iter)

for each search member

Update the position of each member of the population by mathematical

Equation (1.16)

end for

Update $l, a$, and $c$

Evaluate the fitness of all search members

Update $x_{\alpha}, x_{\beta}, x_{\gamma}$, and $x_{\delta}$

$t=t+1$

end while

return $x_{\alpha}$ 
Table 1. Unimodal benchmark functions.

\begin{tabular}{lcccc}
\hline \multicolumn{1}{c}{ Function } & Dim & Range & $\boldsymbol{f}_{\text {min }}$ \\
\hline$F_{1}(x)$ & $=\sum_{i=1}^{n} x_{i}^{2}$ & 30 & {$[-100,100]$} & 0 \\
$F_{2}(x)=\sum_{i=1}^{n}\left|x_{i}\right|+\prod_{i=1}^{n}\left|x_{i}\right|$ & 30 & {$[-10,10]$} & 0 \\
$F_{3}(x)=\sum_{i=1}^{n}\left(\sum_{j-1}^{i} x_{j}\right)^{2}$ & 30 & {$[-100,100]$} & 0 \\
$F_{4}(x)=\max _{i}\left\{\left|x_{i}\right|, 1 \leq i \leq n\right\}$ & 30 & {$[-100,100]$} & 0 \\
$F_{5}(x)=\sum_{i=1}^{n-1}\left[100\left(x_{i+1}-x_{i}^{2}\right)^{2}+\left(x_{i}-1\right)^{2}\right]$ & 30 & {$[-30,30]$} & 0 \\
$F_{6}(x)=\sum_{i=1}^{n}\left(\left[x_{i}+0.5\right]\right)^{2}$ & 30 & {$[-100,100]$} & 0 \\
$F_{7}(x)$ & $=\sum_{i=1}^{n} i x_{i}^{4}+\operatorname{rand}[0,1)$ & 30 & {$[-1.28,1.28]$} & 0 \\
\hline
\end{tabular}

Table 2. Multimodal benchmark functions.

\begin{tabular}{|c|c|c|c|}
\hline Function & Dim & Range & $f_{\min }$ \\
\hline$F_{8}(x)=\sum_{i=1}^{n}-x_{i} \sin \left(\sqrt{\left|x_{i}\right|}\right)$ & 30 & {$[-500,500]$} & $-418.9829 \times 5$ \\
\hline$F_{9}(x)=\sum_{i=1}^{n}\left[x_{i}^{2}-10 \cos \left(2 \pi x_{i}\right)+10\right]$ & 30 & {$[-5.12,5.12]$} & 0 \\
\hline$F_{10}(x)=-20 \exp \left(-0.2 \sqrt{\frac{1}{n} \sum_{i=1}^{n} x_{i}^{2}}\right)-\exp \left(\frac{1}{n} \sum_{i=1}^{n} \cos \left(2 \pi x_{i}\right)\right)+20+e$ & 30 & {$[-32,32]$} & 0 \\
\hline$F_{11}(x)=\frac{1}{4000} \sum_{i=1}^{n} x_{i}^{2}-\prod_{i=1}^{n} \cos \left(\frac{x_{i}}{\sqrt{i}}\right)+1$ & 30 & {$[-600,600]$} & 0 \\
\hline $\begin{aligned} F_{12}(x)= & \frac{\pi}{n}\left\{10 \sin \left(\pi y_{i}\right)+\sum_{i=1}^{n-1}\left(y_{i}-1\right)^{2}\left[1+10 \sin ^{2}\left(\pi y_{i+1}\right)+\left(y_{n-1}\right)^{2}\right]\right\} \\
& +\sum_{i=1}^{n} u\left(x_{i}, 10,100,4\right)\end{aligned}$ & & & \\
\hline \multicolumn{4}{|l|}{$y_{i}=1+\frac{x_{i}+1}{4}$} \\
\hline$\left(k\left(x_{i}-a\right)^{m}\right.$ & & & \\
\hline$u\left(x_{i}, a, k, m\right)= \begin{cases}0 & -a<x_{i}<a \\
k\left(-x_{i}-a\right)^{m} & x_{i}<-a\end{cases}$ & 30 & {$[-50,50]$} & 0 \\
\hline \multicolumn{4}{|l|}{$F_{13}(x)=0.1\left\{\sin ^{2}\left(3 \pi x_{i}\right)+\sum_{i=1}^{n}\left(x_{i}-1\right)^{2}\left[1+\sin ^{2}\left(3 \pi x_{i}+1\right)\right]+\left(x_{n}-1\right)^{2}\right.$} \\
\hline$\left.+\left[1+\sin ^{2}\left(2 \pi x_{n}\right)\right]\right\}+\sum_{i=1}^{n} u\left(x_{i}, 5,100,4\right)$ & 30 & {$[-50,50]$} & 0 \\
\hline
\end{tabular}

\section{Testing functions}

In this section, twenty-three classical functions are used to verify the performance of the MVGWO. These test functions can be divided into three different groups: unimodal, multimodal, and fixed-dimension multimodal functions. Specific details of these functions are represented by Tables 1,2 , and 3 , respectively.

\section{The convergence performance graphs of MVGWO algorithm}

The performances of several population-based meta- heuristics have been verified with the MVGWO variant in order to test the convergence rate, stability, and computational accuracy in a number of iterations in Figure 2. Similar parameter values have been considered for the entire algorithms to make a fair comparison. The results illustrate that, in convergence Figure 2, by plotting the best optimal values of the functions, values have been compared to a number of generations for the simplified model of the molecule with different sizes from 1000 to 5000 dimensions.

The graphs show that the standard test function values quickly decrease as the number of generations increases for newly existing variant solutions as compared 
Table 3. Fixed-dimension multimodal benchmark functions.

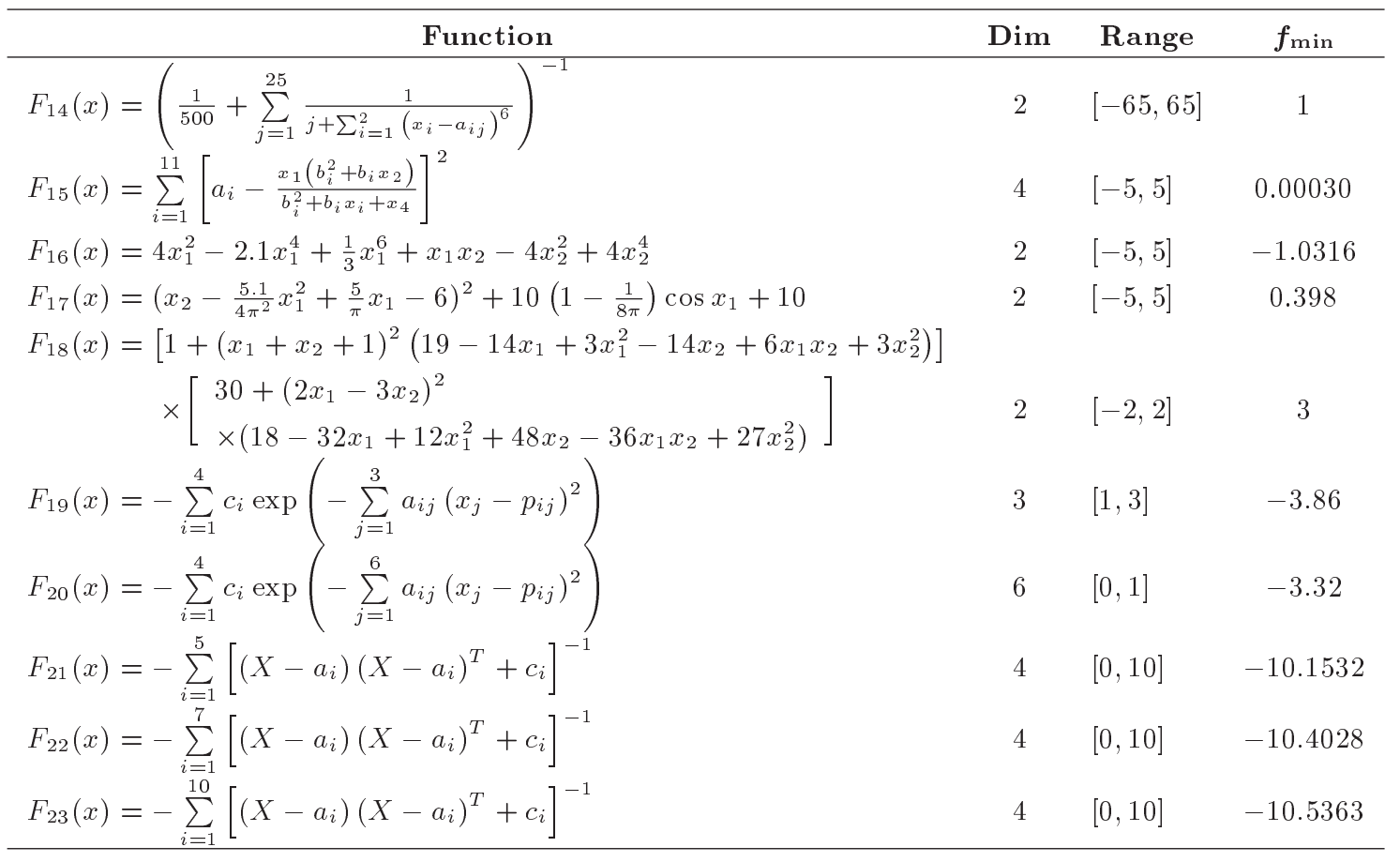
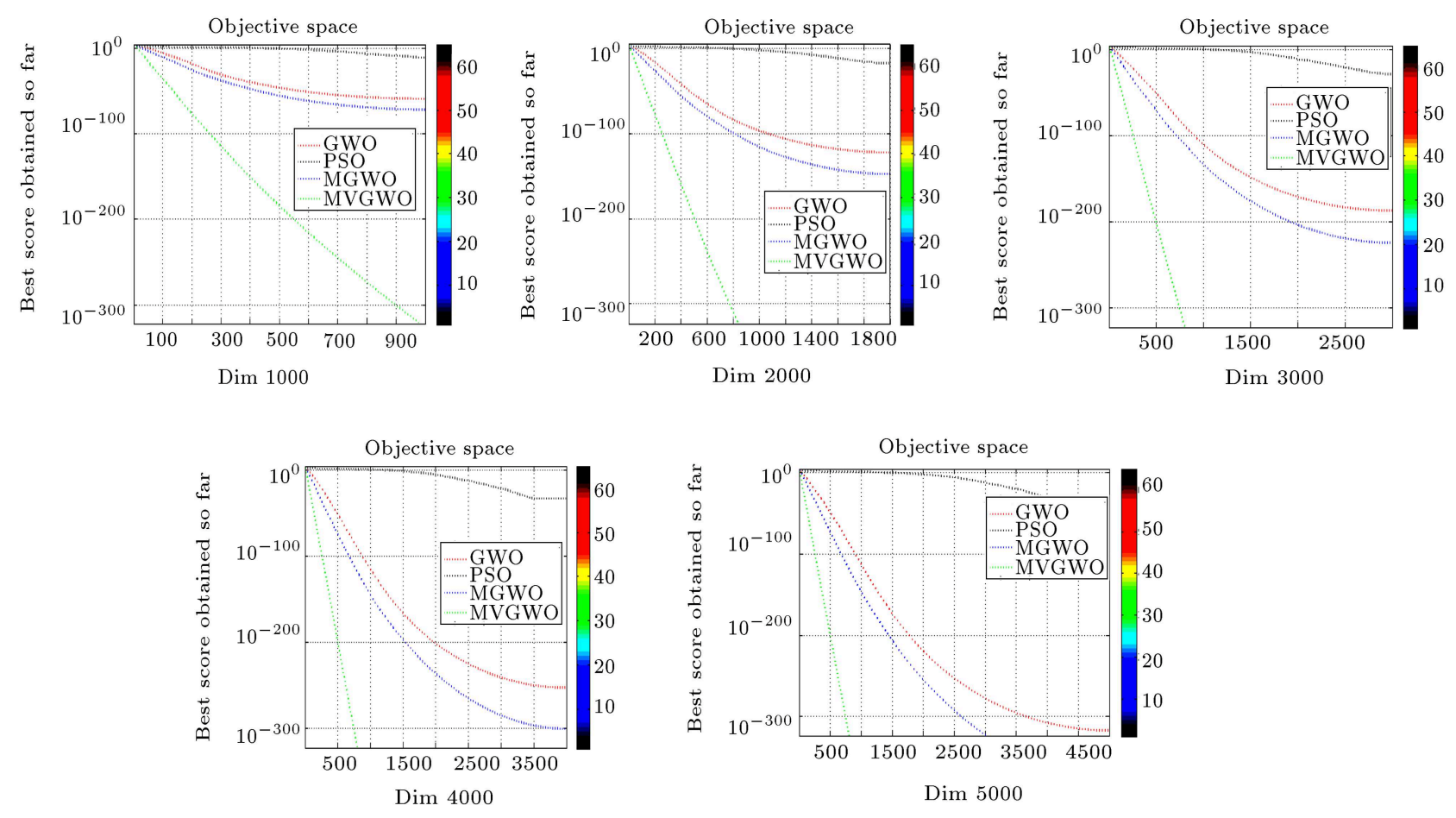

Figure 2. Convergence graphs of algorithms.

to those of the other metaheuristics. In Figure 2, PSO, GWO, HGWO, and MVGWO variants suffer from slow convergence and are stalled in the partitioning procedure; nevertheless, the mean grey wolf variant plays a role for the existing hybrid algorithm to avoid trapping in local minima and accelerate the search.

\section{Results and discussion}

The MVGWO, MGWO, GWO, and PSO algorithms are coded by MATLAB R2013a and implemented by Intel HD Graphics, Pentium-Intel Core (TM), i5 Processor 430 M , 15.6" 16.9 HD LCD, 3GB Memory, 
and 320 GB HDD. Parameters including the number of search agents (30), the maximum number of iterations (1000), and $l \in[2,0]$ are used to confirm the quality of modified meta-heuristics.

Generally, any nature-inspired technique is tested by computing its results with those obtained through other meta-heuristics. In addition, this study follows the same procedure and employs twenty-three classical functions for judgment. These test functions are divided into three parts: unimodal, multimodal, and fixed-dimension multimodal functions. The mathe- matical formulation of classical functions is presented in Tables 1-3. Thirty variables of multimodal and unimodal classical functions are considered to further improve their difficulties.

The accuracy of the newly modified algorithm has been confirmed; thus, this algorithm is applied to the classical, sine dataset, and cantilever beam design functions in terms of minimum objective function values, maximum objective function values, mean, and standard deviation (Tables 4-9).

Herein, the maximum and minimum values of the

Table 4. The optimal solutions obtained by the algorithms on unimodal benchmark functions.

\begin{tabular}{|c|c|c|c|c|c|c|c|c|}
\hline \multirow{2}{*}{$\begin{array}{c}\text { Problem } \\
\text { no. }\end{array}$} & \multicolumn{2}{|c|}{ PSO } & \multicolumn{2}{|c|}{ GWO } & \multicolumn{2}{|c|}{ MGWO } & \multicolumn{2}{|c|}{ MVGWO } \\
\hline & Min & Max & Min & Max & Min & Max & Min & Max \\
\hline 1 & $2.1532 \mathrm{e}-09$ & $6.6032 \mathrm{e}+04$ & $6.1668 \mathrm{e}-61$ & $5.8943 e+04$ & $2.7170 \mathrm{e}-73$ & $6.1504 \mathrm{e}+04$ & 0.00 & $7.1072 \mathrm{e}+04$ \\
\hline 2 & $5.3156 \mathrm{e}-06$ & $1.5784 \mathrm{e}+09$ & $1.9775 \mathrm{e}-35$ & $1.4305 \mathrm{e}+12$ & $7.4227 \mathrm{e}-43$ & $3.2669 \mathrm{e}+09$ & $1.6053 \mathrm{e}-175$ & $3.7809 e+13$ \\
\hline 3 & 12.0227 & $1.9267 \mathrm{e}+05$ & $3.3073 \mathrm{e}-15$ & $1.8876 \mathrm{e}+05$ & $2.5227 \mathrm{e}-25$ & $1.1651 \mathrm{e}+05$ & $1.2105 \mathrm{e}-282$ & $6.1749 e+04$ \\
\hline 4 & 2.6791 & 0.6791 & $2.6647 \mathrm{e}-14$ & 89.0706 & $2.4202 \mathrm{e}-20$ & 88.0388 & $1.7751 \mathrm{e}-152$ & 91.6933 \\
\hline 5 & 107.7967 & 107.7967 & 27.1178 & $2.1621 \mathrm{e}+08$ & 27.1631 & $2.3050 \mathrm{e}+08$ & 27.1003 & $3.1495 e+08$ \\
\hline 6 & $2.6127 \mathrm{e}-11$ & $6.8467 \mathrm{e}+04$ & 1.2547 & $6.5240 \mathrm{e}+04$ & 1.2505 & $7.0794 \mathrm{e}+04$ & 3.8767 & $7.4148 e+04$ \\
\hline 7 & 0.0367 & 133.3527 & $3.6149 \mathrm{e}-04$ & 92.2196 & $5.7914 \mathrm{e}-04$ & 80.6522 & $1.4141 \mathrm{e}-05$ & 153.5907 \\
\hline
\end{tabular}

Table 5. The statistical results obtained by the algorithms on unimodal benchmark functions.

\begin{tabular}{|c|c|c|c|c|c|c|c|c|}
\hline \multirow{2}{*}{$\begin{array}{c}\text { Problem } \\
\text { no. }\end{array}$} & \multicolumn{2}{|c|}{ PSO } & \multicolumn{2}{|c|}{ GWO } & \multicolumn{2}{|c|}{ MGWO } & \multicolumn{2}{|c|}{ MVGWO } \\
\hline & $\mu$ & $\sigma$ & $\mu$ & $\sigma$ & $\mu$ & $\sigma$ & $\mu$ & $\sigma$ \\
\hline 1 & 408.5162 & $3.9524 \mathrm{e}+03$ & 215.8357 & $2.5750 \mathrm{e}+03$ & 173.1716 & $2.5391 \mathrm{e}+03$ & 125.5010 & $2.4662 \mathrm{e}+03$ \\
\hline 2 & $9.5933 \mathrm{e}+06$ & $3.0290 \mathrm{e}+08$ & $1.4316 \mathrm{e}+09$ & $4.5236 \mathrm{e}+10$ & $3.2708 \mathrm{e}+06$ & $1.0331 \mathrm{e}+08$ & $1.2715 e+10$ & $1.1956 e+12$ \\
\hline 3 & $1.8412 \mathrm{e}+03$ & $1.3960 \mathrm{e}+04$ & $1.7745 \mathrm{e}+03$ & $1.0505 \mathrm{e}+04$ & 674.7361 & $5.8039 e+03$ & $1.2536 e+03$ & $1.0787 e+04$ \\
\hline 4 & 3.5511 & 7.3907 & 2.6146 & 12.1387 & 0.9451 & 6.7658 & 0.3492 & 4.3699 \\
\hline 5 & $5.9195 \mathrm{e}+05$ & $1.0872 \mathrm{e}+07$ & $7.4518 \mathrm{e}+05$ & $1.0897 \mathrm{e}+07$ & $6.7132 \mathrm{e}+05$ & $9.9844 \mathrm{e}+06$ & $3.5409 e+05$ & $1.0001 \mathrm{e}+07$ \\
\hline 6 & 516.2407 & $4.6252 \mathrm{e}+03$ & 335.9674 & $3.4849 \mathrm{e}+03$ & 197.7060 & $2.8166 \mathrm{e}+03$ & 123.8129 & $2.5129 e+03$ \\
\hline 7 & 53.3529 & 56.2679 & 0.4942 & 5.8041 & 0.1335 & 2.6681 & 0.2020 & 4.9747 \\
\hline
\end{tabular}

Table 6. The optimal solutions obtained by the algorithms on multimodal benchmark functions.

\begin{tabular}{|c|c|c|c|c|c|c|c|c|}
\hline \multirow{2}{*}{$\begin{array}{c}\text { Problem } \\
\text { no. }\end{array}$} & \multicolumn{2}{|c|}{ PSO } & \multicolumn{2}{|c|}{ GWO } & \multicolumn{2}{|c|}{ MGWO } & \multicolumn{2}{|c|}{ MVGWO } \\
\hline & Min & Max & Min & Max & Min & Max & Min & Max \\
\hline 1 & $-6.6067 e+03$ & $-1.4706 e+03$ & $-5.8056 \mathrm{e}+03$ & $-2.4436 \mathrm{e}+03$ & $-4.8344 e+03$ & $-2.5399 \mathrm{e}+03$ & $-2.2540 e+03$ & $-2.2373 e+03$ \\
\hline 2 & 39.7987 & 422.6854 & $5.6843 \mathrm{e}-14$ & 458.7865 & 0 & 438.1148 & 0 & 488.0757 \\
\hline 3 & $1.5846 \mathrm{e}-05$ & 20.5268 & $1.5099 \mathrm{e}-14$ & 20.7623 & $1.5099 \mathrm{e}-14$ & 20.5150 & $1.4409 e-15$ & 20.8472 \\
\hline 4 & $2.0755 \mathrm{e}-12$ & 667.1103 & 0.0092 & 665.7767 & 0 & 527.3462 & 0 & 555.0353 \\
\hline 5 & $2.0193 \mathrm{e}-12$ & $6.1692 \mathrm{e}+08$ & 0.0304 & $5.5204 \mathrm{e}+08$ & 0.0538 & $6.1414 \mathrm{e}+08$ & 0.5589 & $8.1057 e+08$ \\
\hline 6 & $6.5797 \mathrm{e}-08$ & $1.0597 \mathrm{e}+09$ & 0.6975 & $8.0560 \mathrm{e}+08$ & 1.1284 & $9.0172 \mathrm{e}+08$ & 0.1930 & $9.2376 e+08$ \\
\hline
\end{tabular}

Table 7. The statistical results obtained by the algorithms on multimodal benchmark functions.

\begin{tabular}{|c|c|c|c|c|c|c|c|c|}
\hline \multirow{2}{*}{$\begin{array}{c}\text { Problem } \\
\text { No. }\end{array}$} & \multicolumn{2}{|c|}{ PSO } & \multicolumn{2}{|c|}{ GWO } & \multicolumn{2}{|c|}{ MGWO } & \multicolumn{2}{|c|}{ MVGWO } \\
\hline & $\mu$ & $\sigma$ & $\mu$ & $\sigma$ & $\mu$ & $\sigma$ & $\mu$ & $\sigma$ \\
\hline 8 & $-6.0956 \mathrm{e}+03$ & $1.1171 \mathrm{e}+03$ & $-4.0393 e+03$ & 967.7908 & $-3.3352 \mathrm{e}+03$ & 731.9012 & $-2.2511 e+03$ & 6.3372 \\
\hline 9 & 161.3787 & 124.3555 & 10.7854 & 48.0111 & 4.9465 & 32.6783 & 2.3389 & 26.4361 \\
\hline 10 & 2.9657 & 3.4941 & 0.4125 & 2.2762 & 0.2497 & 1.7307 & 0.1045 & 1.1726 \\
\hline 11 & 25.7028 & 100.2551 & 3.1497 & 32.9323 & 1.4174 & 20.4885 & 0.9590 & 18.9328 \\
\hline 12 & $1.1219 \mathrm{e}+06$ & $2.2349 \mathrm{e}+07$ & $1.5736 \mathrm{e}+06$ & $2.4904 \mathrm{e}+07$ & $1.1003 \mathrm{e}+06$ & $2.1982 \mathrm{e}+07$ & $1.0443 e+06$ & $2.1584 e+07$ \\
\hline 13 & $2.5209 \mathrm{e}+06$ & $4.4037 \mathrm{e}+07$ & $3.2041 \mathrm{e}+06$ & $4.3667 \mathrm{e}+07$ & $1.5261 \mathrm{e}+06$ & $3.2837 \mathrm{e}++07$ & $1.1030 \mathrm{e}+06$ & $2.9672 \mathrm{e}+07$ \\
\hline
\end{tabular}


Table 8. The optimal solutions obtained by the algorithms on fixed-dimension multimodal benchmark functions.

\begin{tabular}{|c|c|c|c|c|c|c|c|c|}
\hline \multirow{2}{*}{$\begin{array}{c}\text { Problem } \\
\text { no. }\end{array}$} & \multicolumn{2}{|c|}{ PSO } & \multicolumn{2}{|c|}{ GWO } & \multicolumn{2}{|c|}{ MGWO } & \multicolumn{2}{|c|}{ MVGWO } \\
\hline & Min & Max & Min & Max & Min & $\operatorname{Max}$ & Min & Max \\
\hline 7 & 2.9920 & 12.6709 & 10.7632 & 86.5835 & 12.6705 & 76.5329 & 2.9821 & 35.7641 \\
\hline 8 & $9.8869 \mathrm{e}-04$ & 0.3069 & $3.0750 \mathrm{e}-04$ & 0.1331 & $3.0749 \mathrm{e}-04$ & 0.2142 & $3.749 \mathrm{e}-04$ & 0.6090 \\
\hline 9 & -1.0316 & 0.0804 & -1.0316 & -0.1653 & -1.0316 & -0.8485 & -1.0316 & -0.8485 \\
\hline 10 & 0.3979 & 2.2225 & 0.3979 & 0.4187 & 0.3979 & 0.4716 & 0.3979 & 2.5346 \\
\hline 11 & 3 & 73.9801 & 3 & 44.4885 & 3 & 58.2138 & 3 & 171.6938 \\
\hline 12 & -3.8628 & -3.6393 & -3.8596 & -3.6339 & -3.8627 & -2.9834 & -3.8628 & -3 \\
\hline 13 & -3.3220 & -0.7636 & -2.8404 & -2.1889 & -3.1421 & -1.7246 & -3.2450 & -1.2158 \\
\hline 14 & -10.1532 & -10.1532 & -5.0552 & -0.3997 & -5.0999 & -0.3774 & -5.0999 & -0.3282 \\
\hline 15 & -5.1288 & -1.2345 & -10.4024 & -0.6547 & -10.4022 & -0.5490 & -10.6466 & -0.4647 \\
\hline 16 & -10.5364 & -0.6398 & -10.5360 & -0.7648 & -10.5357 & -0.7656 & -10.9786 & -0.6271 \\
\hline
\end{tabular}

Table 9. The statistical results obtained by the algorithms on fixed-dimension multimodal benchmark functions.

\begin{tabular}{|c|c|c|c|c|c|c|c|c|}
\hline \multirow{2}{*}{$\begin{array}{c}\text { Problem } \\
\text { no. }\end{array}$} & \multicolumn{2}{|c|}{ PSO } & \multicolumn{2}{|c|}{ GWO } & \multicolumn{2}{|c|}{ MGWO } & \multicolumn{2}{|c|}{ MVGWO } \\
\hline & $\mu$ & $\sigma$ & $\mu$ & $\sigma$ & $\mu$ & $\sigma$ & $\mu$ & $\sigma$ \\
\hline 14 & 2.1505 & 0.9110 & 11.2055 & 4.2682 & 12.9194 & 2.8310 & 3.0536 & 1.4674 \\
\hline 15 & 0.0016 & 0.0107 & $8.6397 \mathrm{e}-04$ & 0.0053 & $7.8362 \mathrm{e}-04$ & 0.0070 & 0.0013 & 0.0201 \\
\hline 16 & -1.0299 & 0.0363 & -1.0275 & 0.1167 & -1.0303 & 0.0287 & -1.0312 & 0.0061 \\
\hline 17 & 0.4004 & 0.0584 & 0.3992 & 0.0041 & 0.4004 & 0.0074 & 0.4012 & 0.0707 \\
\hline 18 & 3.2555 & 2.4028 & 3.1319 & 2.0225 & 3.1918 & 2.5571 & 3.0323 & 0.6594 \\
\hline 19 & $-3 . .8614$ & 0.0077 & -3.8555 & 0.0147 & -3.8586 & 0.0282 & -3.8565 & 0.0129 \\
\hline 20 & -3.1235 & 0.2311 & -2.8158 & 0.0565 & -3.1068 & 0.0769 & -3.2015 & 0.1366 \\
\hline 21 & -4.2193 & 2.2680 & -4.7394 & 0.7968 & -2.9990 & 1.2537 & -4.9287 & 0.3697 \\
\hline 22 & -4.8911 & 0.6718 & -7.7375 & 2.6723 & -6.6836 & 3.4455 & -8.6227 & 0.4368 \\
\hline 23 & -9.3903 & 2.5222 & -8.2760 & 1.8966 & -7.7390 & 2.8551 & -4.8748 & 0.3978 \\
\hline
\end{tabular}

objective functions produce the best suitable cost of the classical problems in the least number of iterations. On the other hand, the mean and standard deviation of statistical values are used to evaluate the reliability. Further, the convergence graphs of the classical problems represent the convergence performance of the variants.

Tables 4, 6, and 8 show that the newly modified algorithm produces the best optimal values of the classical problems in terms of minimum and maximum values of the functions as compared to other metaheuristics. Tables 5, 7, and 9 illustrate that the modified algorithm produces the superior quality of standard and mean values on the maximum classical functions in the form of the least values as compared to other meta-heuristics. In the end, the convergence graphs (Figures 3-25) prove that the existing approach finds the best possible optimal values of the standard functions in the least number of iterations as compared to others.

Based on the results given in Tables 4 and 5 , it is clear that the proposed variant outperforms other

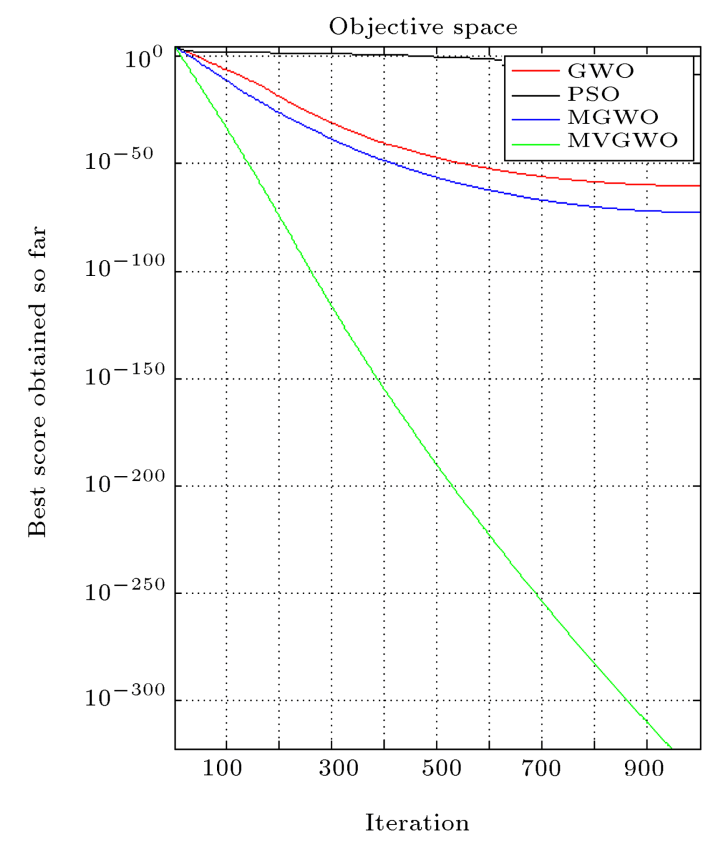

Figure 3. Convergence graph of fixed-dimension multimodal benchmark function $\left(F_{1}\right)$. 


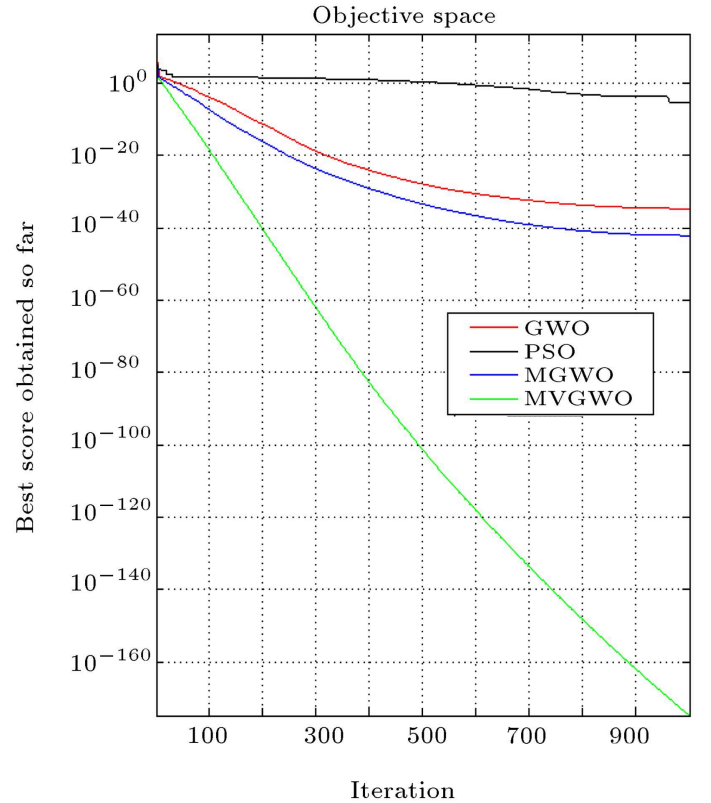

Figure 4. Convergence graph of fixed-dimension multimodal benchmark function $\left(F_{2}\right)$.

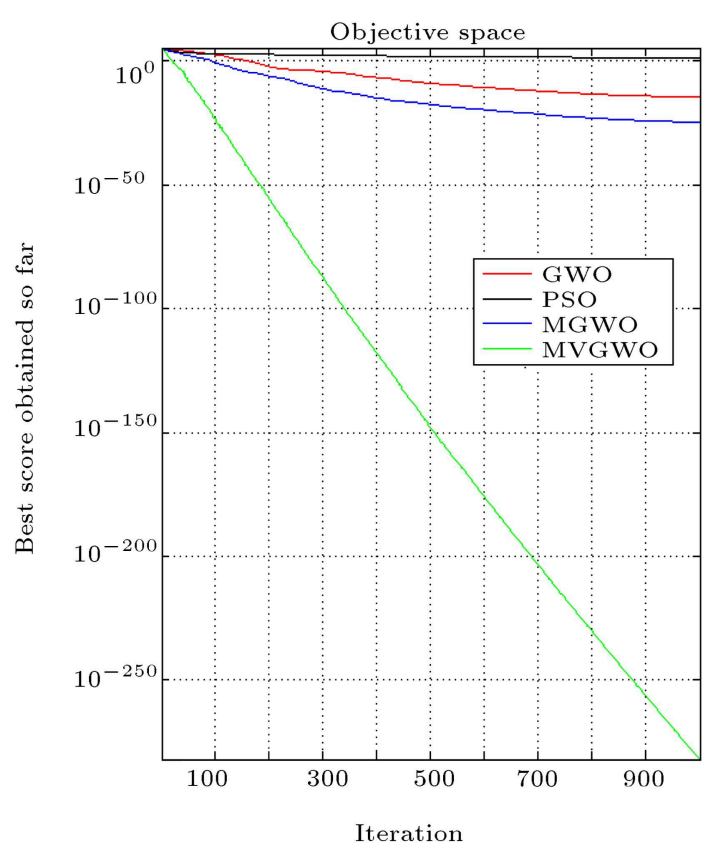

Figure 5. Convergence graph of fixed-dimension multimodal benchmark function $\left(F_{3}\right)$.

meta-heuristics, including PSO, GWO, and MGWO, in terms of mean, standard deviation, and $\min / \max$ cost function, and exploits the optimum. Accordingly, the proposed variant is highly comparable to other metaheuristics.

Further, the convergence behaviors of the proposed variant, PSO, MGWO, and GWO algorithms have been investigated, and convergence curve is plotted in Figures 3-25. In order to examine the convergence behavior of the modified variant of GWO,

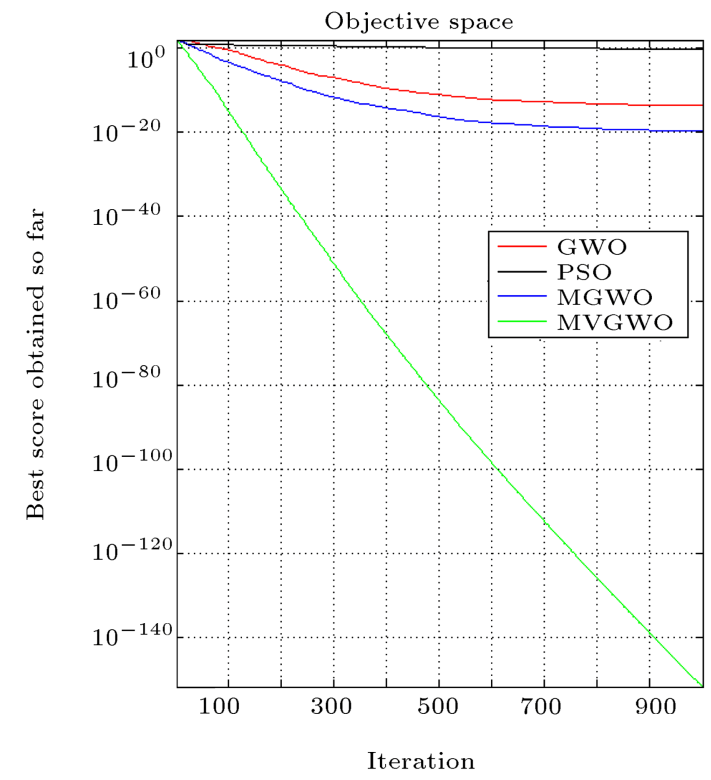

Figure 6. Convergence graph of fixed-dimension multimodal benchmark function $\left(F_{4}\right)$.

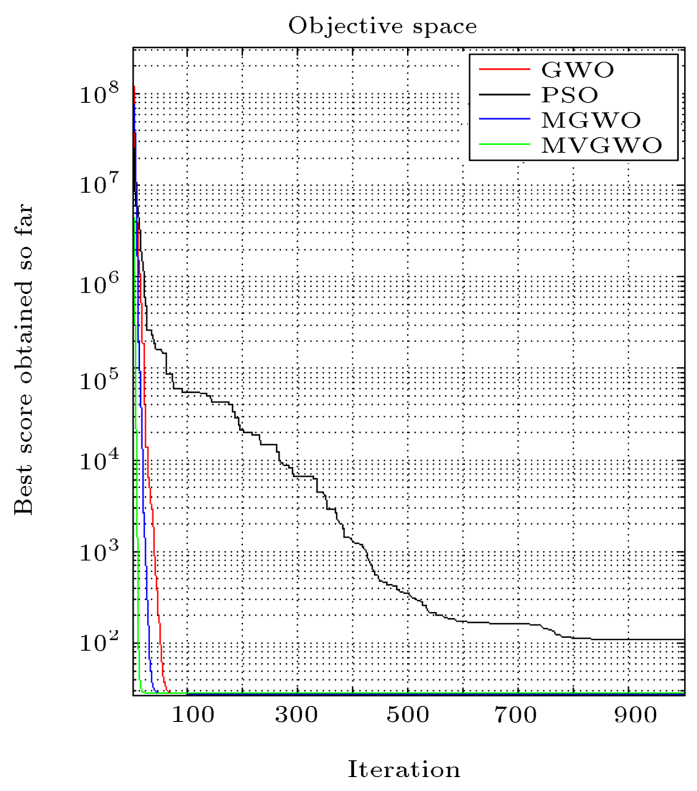

Figure 7. Convergence graph of fixed-dimension multimodal benchmark function $\left(F_{5}\right)$.

PSO, MGWO, and grey wolf optimizer algorithms, the search history and path of the first search member of the population in its first dimension are illustrated in Figures 3-25. Based on the convergence curve, it is observed that the modified variant produces better convergence points as compared to others.

Furthermore, the appropriate results of multimodal and fixed-dimension multimodal functions are illustrated in Tables 6-9. The multimodal and fixeddimension functions have many local optima whose number and dimension grow exponentially. This makes them fit for benchmarking the exploration capacity 


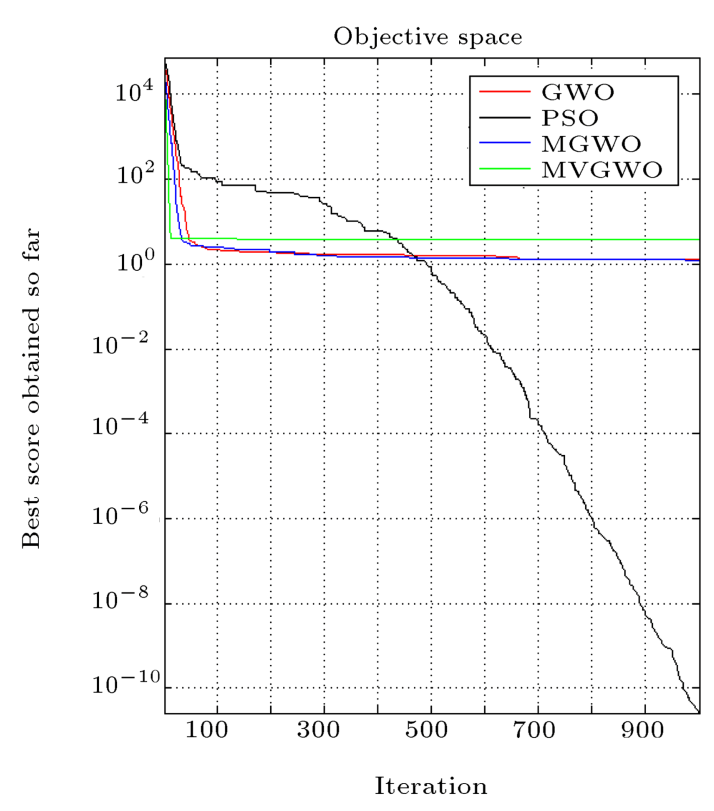

Figure 8. Convergence graph of fixed-dimension multimodal benchmark function $\left(F_{6}\right)$.

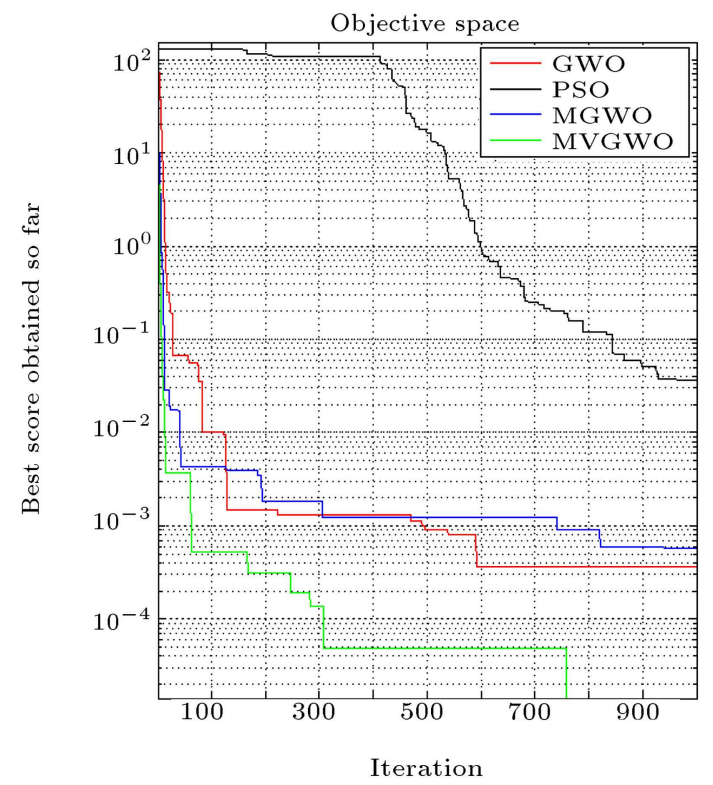

Figure 9. Convergence graph of fixed-dimension multimodal benchmark function $\left(F_{7}\right)$.

of a variant. Based on the results of Tables 6-9, the modified variant is able to present better solution quality with respect to the maximum number of multimodal and fixed-dimension multi-modal functions as compared to PSO, GWO, and MGWO algorithm. These solutions demonstrate that the MVGWO has advantages in terms of exploration.

A number of criteria have been used to determine the accuracy of the proposed algorithm, GWO, PSO, and MGWO. The mean and standard deviation of statistical values are used to evaluate reliability in Tables 5, 7, and 9. The average computation time of

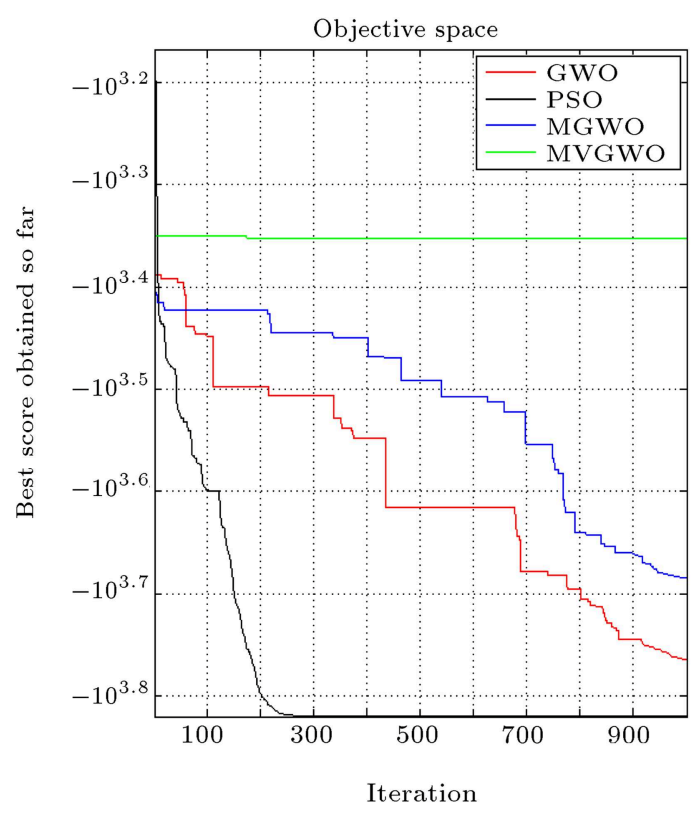

Figure 10. Convergence graph of fixed-dimension multimodal benchmark function $\left(F_{8}\right)$.

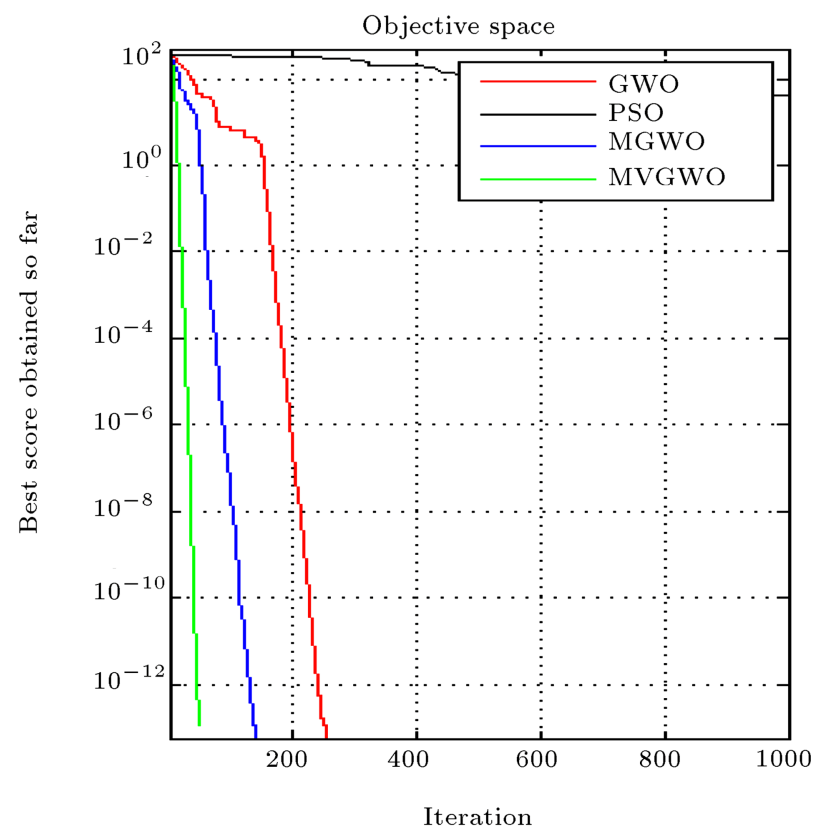

Figure 11. Convergence graph of fixed-dimension multimodal benchmark function $\left(F_{9}\right)$.

the successful runs and the average number of function evaluations of successful runs are applied to estimate the cost of the standard function.

In Figures 3-25, the convergence performances of GWO, PSO, MGWO, and MVGWO algorithms in solving classical problems are compared. The obtained convergence solutions prove that the MVGWO algorithm is more capable to find the best optimal solution in the minimum number of iterations. Hence, MVGWO algorithm avoids premature convergence of the search 


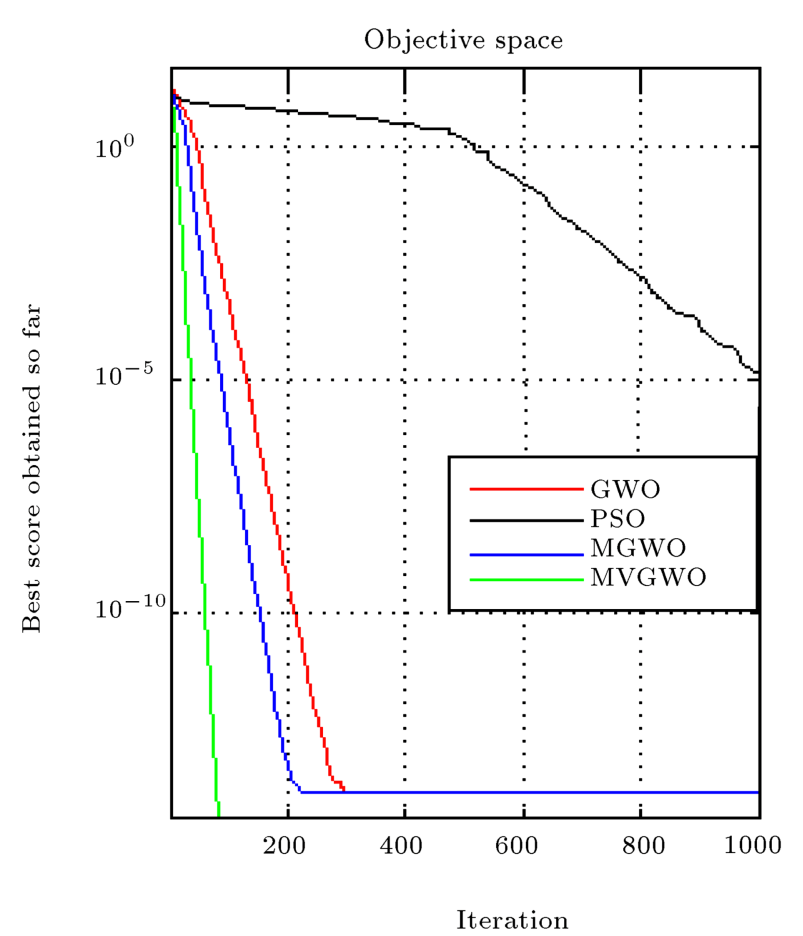

Figure 12. Convergence graph of fixed-dimension multimodal benchmark function $\left(F_{10}\right)$

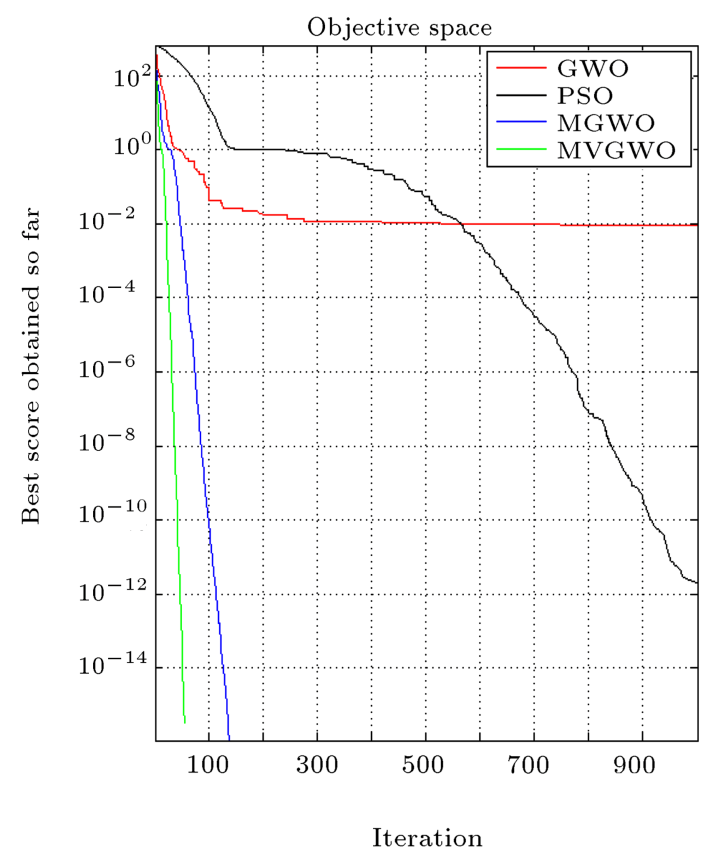

Figure 13. Convergence graph of fixed-dimension multimodal benchmark function $\left(F_{11}\right)$.

process to local optimal point and provides a superior exploration of the search course.

To sum up, based on all of the simulation solutions, the recent existing algorithm is very helpful in increasing the efficiency of the GWO algorithm in terms of result quality and computational efforts.

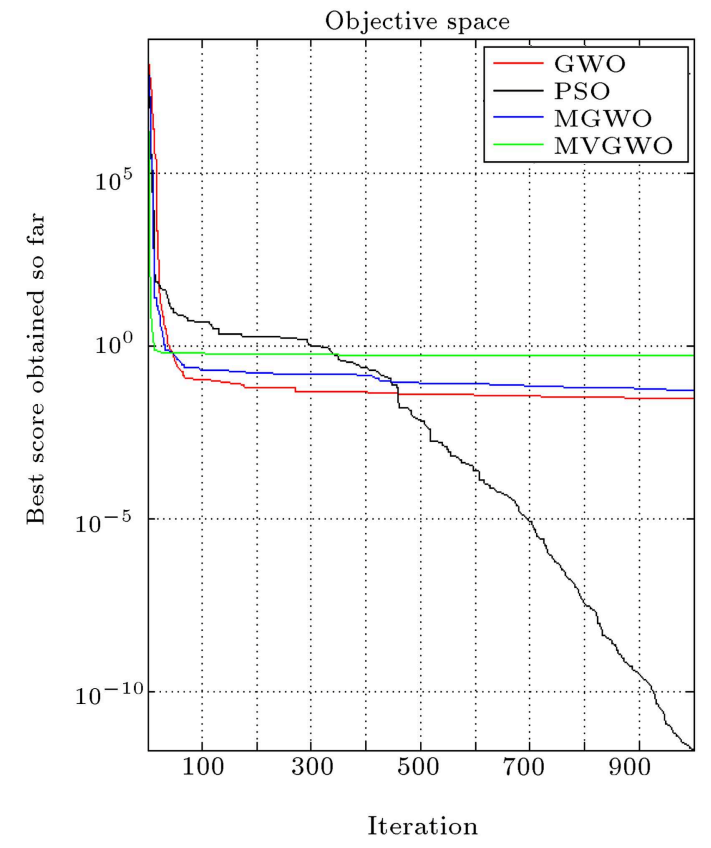

Figure 14. Convergence graph of fixed-dimension multimodal benchmark function $\left(F_{12}\right)$.

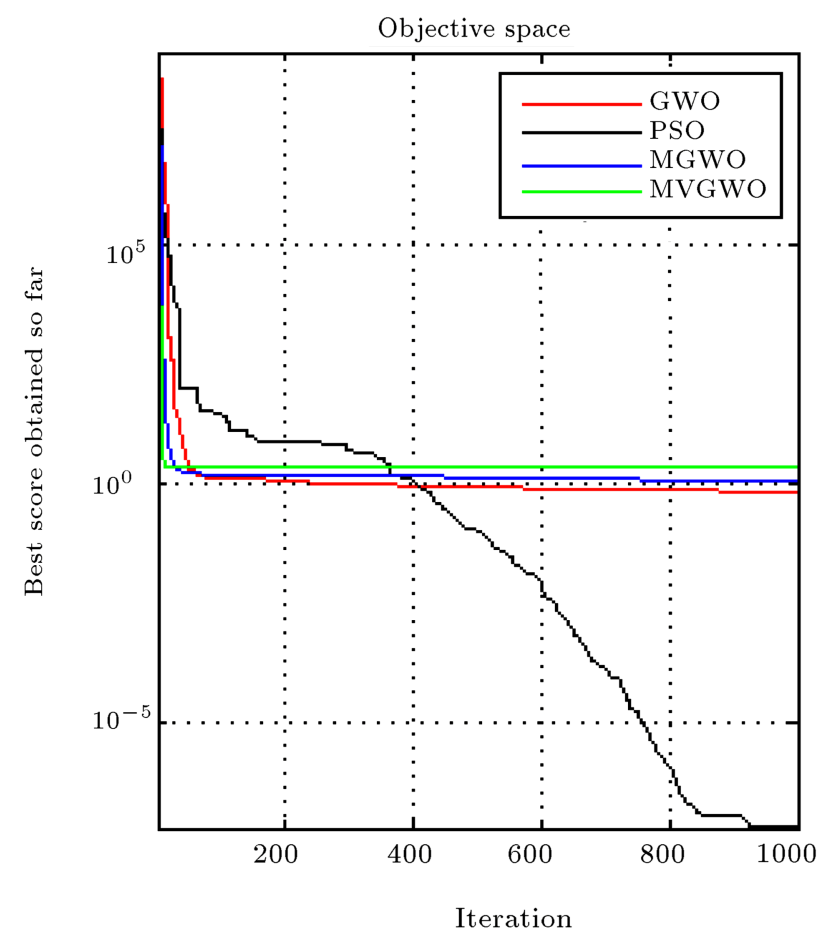

Figure 15. Convergence graph of fixed-dimension multimodal benchmark function $\left(F_{13}\right)$.

\section{Sine dataset function}

This dataset has a number of attributes 01 and structures 1-15-1 chosen to train and solve this dataset [30]. This function has four peaks that make it extremely difficult to approximate. Sine dataset function has been tested on different nature-inspired meta-heuristics. Ac- 


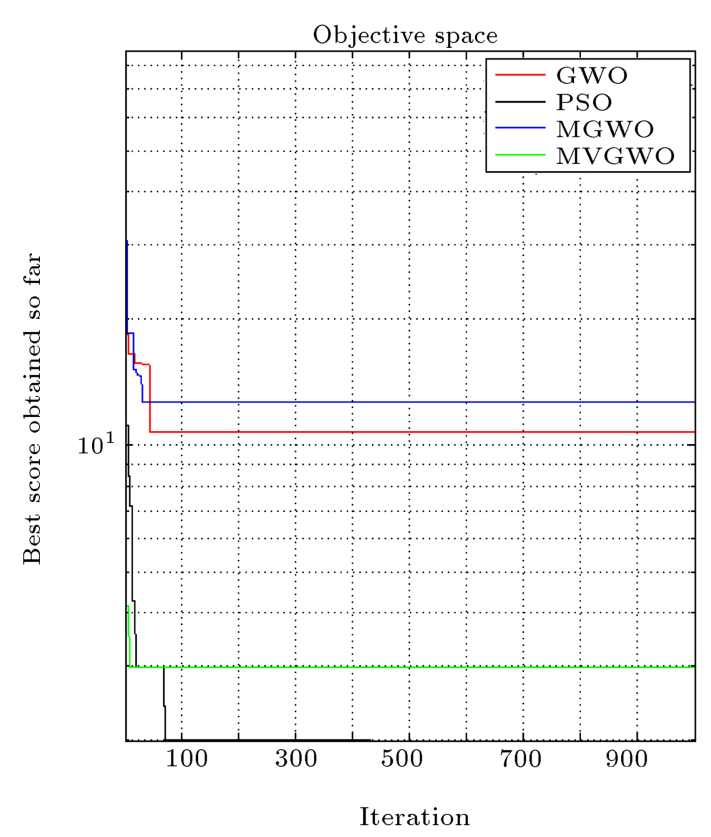

Figure 16. Convergence graph of fixed-dimension multimodal benchmark function $\left(F_{14}\right)$.

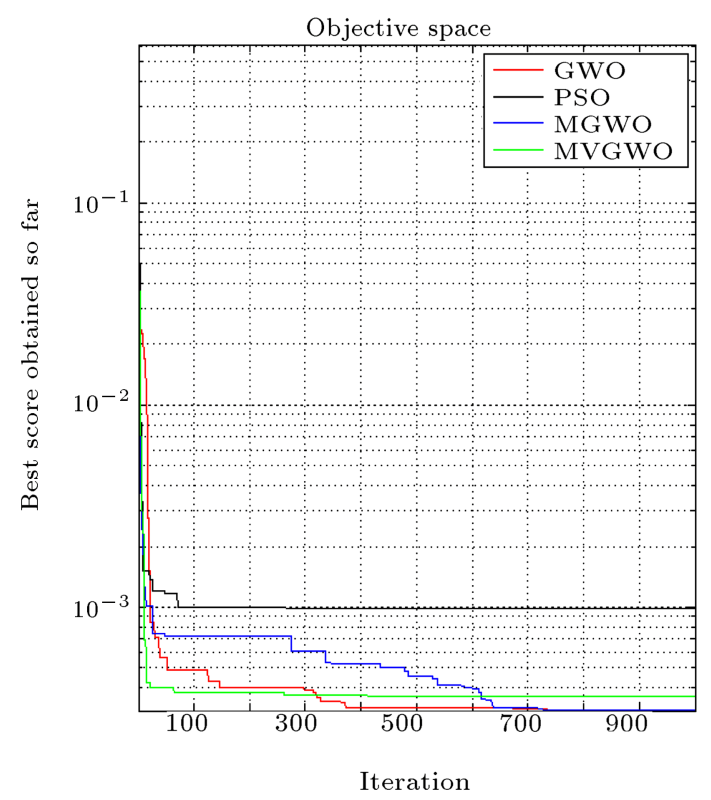

Figure 17. Convergence graph of fixed-dimension multimodal benchmark function $\left(F_{15}\right)$.

cording to the obtained results, it is observed that the modified variant of grey wolf optimizer provides extremely accurate solutions in this dataset as can be inferred from test error in Table 10, and convergence and best solution performance of MVGWO are plotted in Figures 26 and 27.

\section{Cantilever beam design function}

This function is associated with the design variables including the width of different beam elements, weight

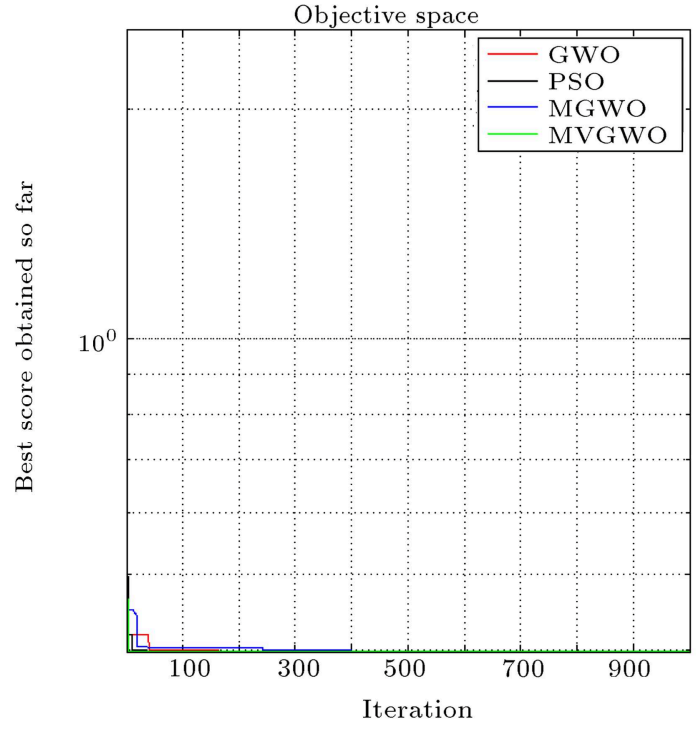

Figure 18. Convergence graph of fixed-dimension multimodal benchmark function $\left(F_{16}\right)$.

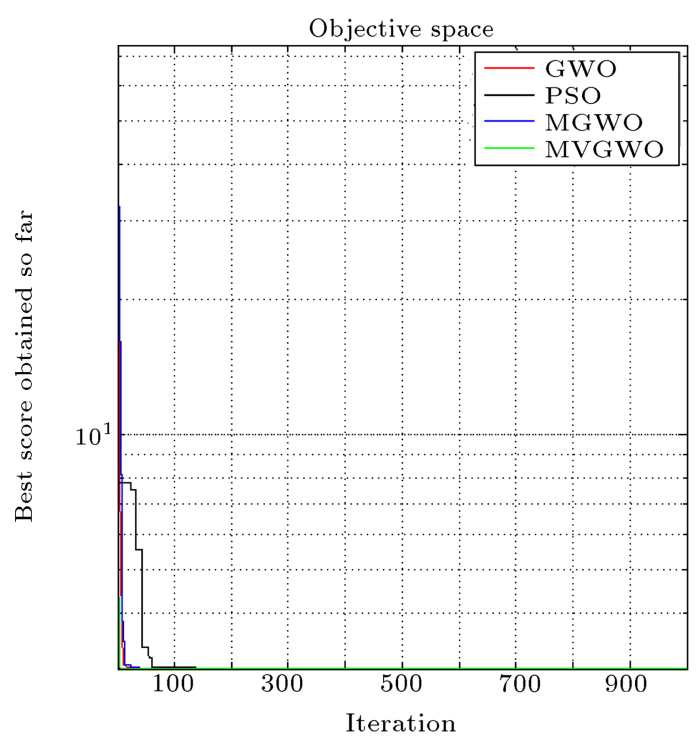

Figure 19. Convergence graph of fixed-dimension multimodal benchmark function $\left(F_{17}\right)$.

Table 10. Experimental results for the sine datasets.

\begin{tabular}{lccc}
\hline Algorithm & $\boldsymbol{\mu}$ & $\boldsymbol{\sigma}$ & Test error \\
\hline MVGWO & 0.2549 & 0.0018 & $2.7221 \mathrm{e}+04$ \\
GWO & 0.261970 & 0.115080 & 43.754 \\
PSO & 0.526530 & 0.072876 & 124.89 \\
GA & 0.421070 & 0.061206 & 111.25 \\
ACO & 0.529830 & 0.053305 & 117.71 \\
ES & 0.706980 & 0.077409 & 142.31 \\
PBIL & 0.483340 & 0.007935 & 149.60 \\
\hline
\end{tabular}

optimization, and constant thickness [51]. A quick description of the cantilever beam function is presented as follows:

$$
\min (X)=0.0624(l+m+n+o+p),
$$




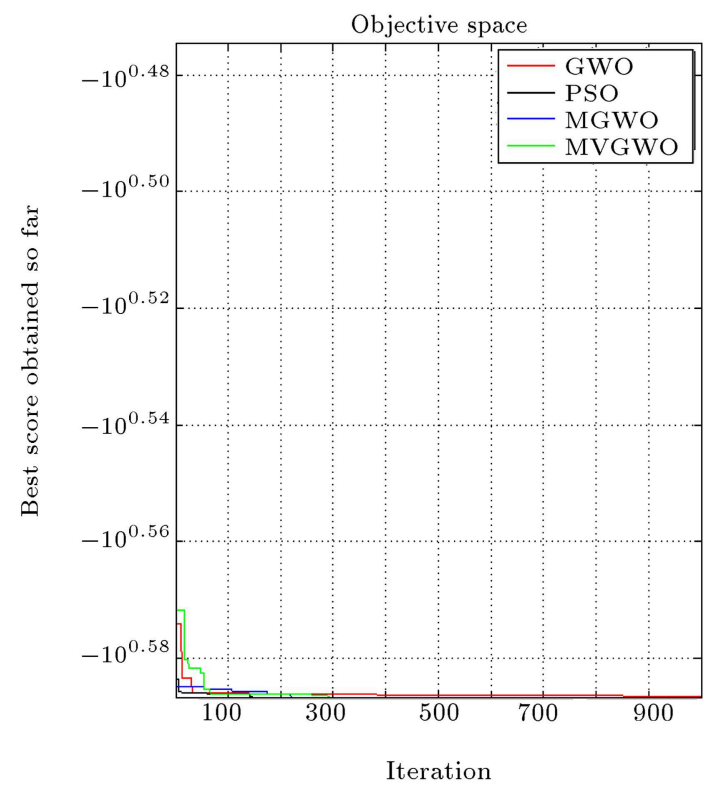

Figure 20. Convergence graph of fixed-dimension multimodal benchmark function $\left(F_{18}\right)$.

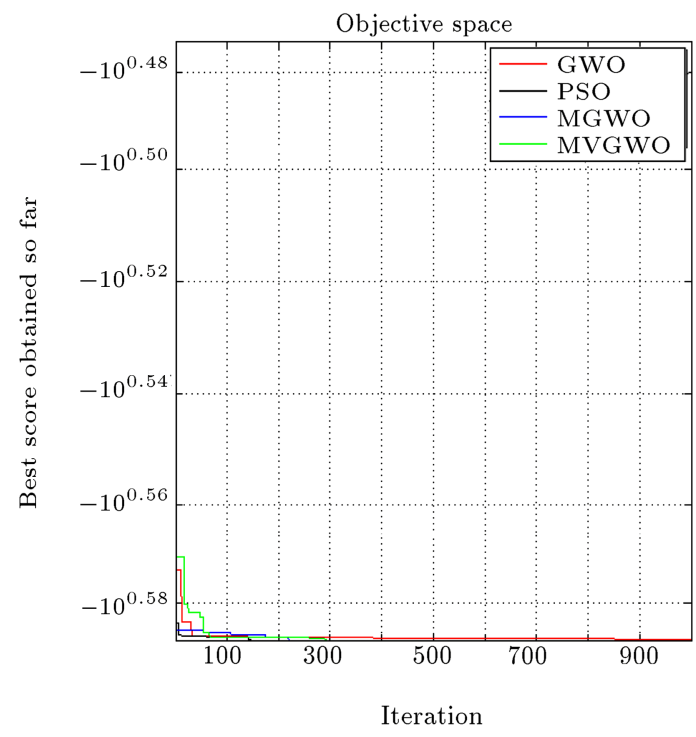

Figure 21. Convergence graph of fixed-dimension multimodal benchmark function $\left(F_{19}\right)$.

subject to:

$$
g(X)=\frac{61}{l^{3}}+\frac{37}{m^{3}}+\frac{19}{n^{3}}+\frac{7}{o^{3}}+\frac{1}{p^{3}}-1 \leq 0
$$

where $0.01 \leq l, m, n, o, p \leq 100$. The global optimal results of the given function are listed in Table 11 .

During the last few decades, several researchers have used different types of meta-heuristics to find the best possible optimal solutions for the cantilever beam design function in the literature such as convex linearization method (CONLIN) [51], CS [51], method of moving asymptotes (MMA) [51], Grid-based Clustering

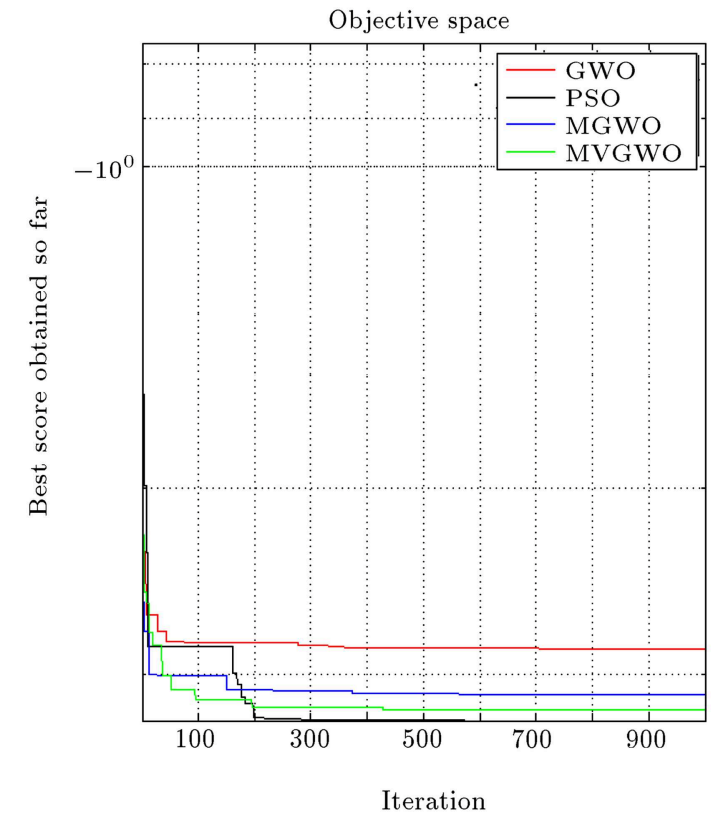

Figure 22. Convergence graph of fixed-dimension multimodal benchmark function $\left(F_{20}\right)$.

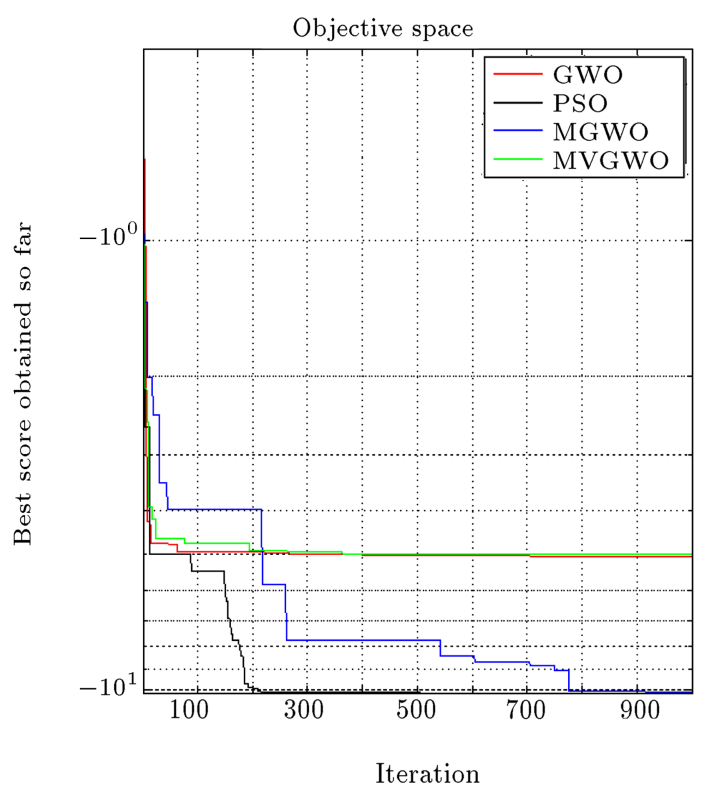

Figure 23. Convergence graph of fixed-dimension multimodal benchmark function $\left(F_{21}\right)$.

Algorithms-I and II (GCA-I and GCA-II) [52], and Symbiotic Organisms Search (SOS) [53].

The experimental results of different variants for the given function are illustrated in Table 11. That experiment has been tested on the following parameter settings: search agents (30) and the maximum number of iterations (500).

It can be seen that the best optimal value of the cantilever beam design function on MVGWO is 1.33966. Hence, MVGWO variant gives better quality of the solutions as compared to other recent algorithms. 


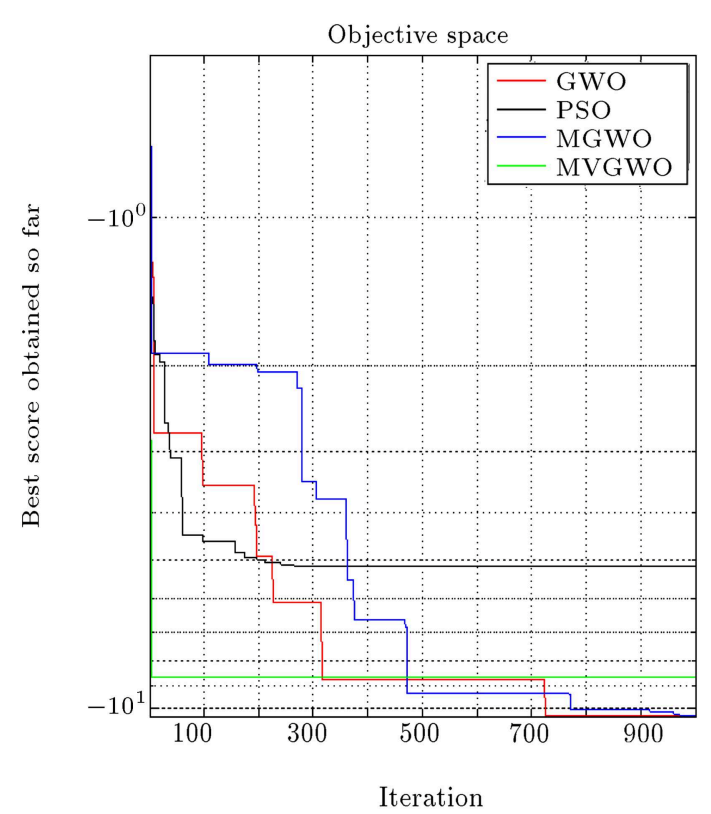

Figure 24. Convergence graph of fixed-dimension multimodal benchmark function $\left(F_{22}\right)$.

\section{Conclusion}

This paper presented a Modified Variant of Grey Wolf Optimization called MVGWO. This modified variant was developed by modifying the encircling behavior and the position update equation of Grey Wolf Optimization (GWO) algorithm with the aim of improving the performance, convergence speed, and accuracy of the GWO meta-heuristics. These modifications were used to make a balance between exploration and exploration over the path of generations. The performance of the proposed variant was tested using several benchmark functions. It was observed that the modified variant had the edge of high exploration over other meta-heuristics such as Particle Swarm Optimization (PSO), GWO, and Mean Grey Wolf Optimization (MGWO).

Further, the performance of the modified variant was tested on sine dataset and cantilever beam design functions. Moreover, the experimental results

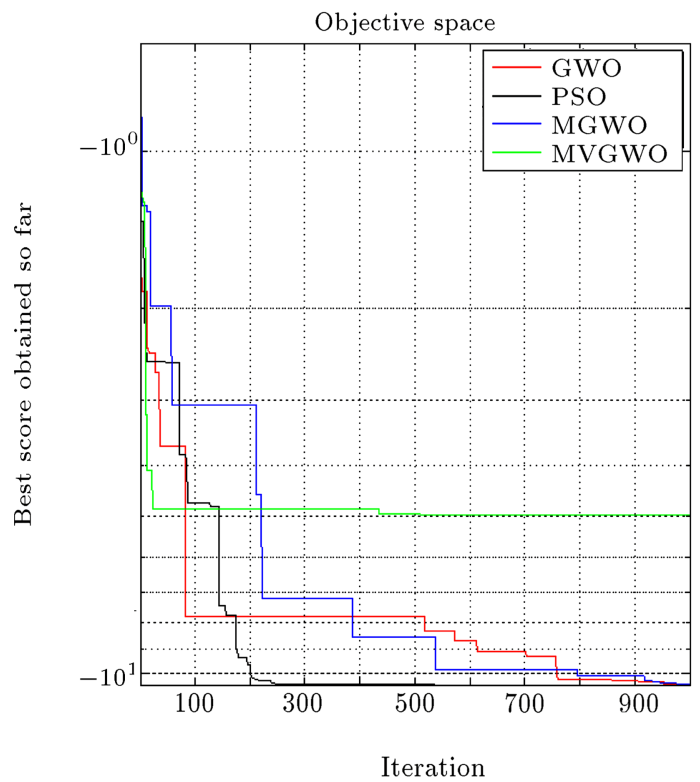

Figure 25. Convergence graph of fixed-dimension multimodal benchmark function $\left(F_{23}\right)$.

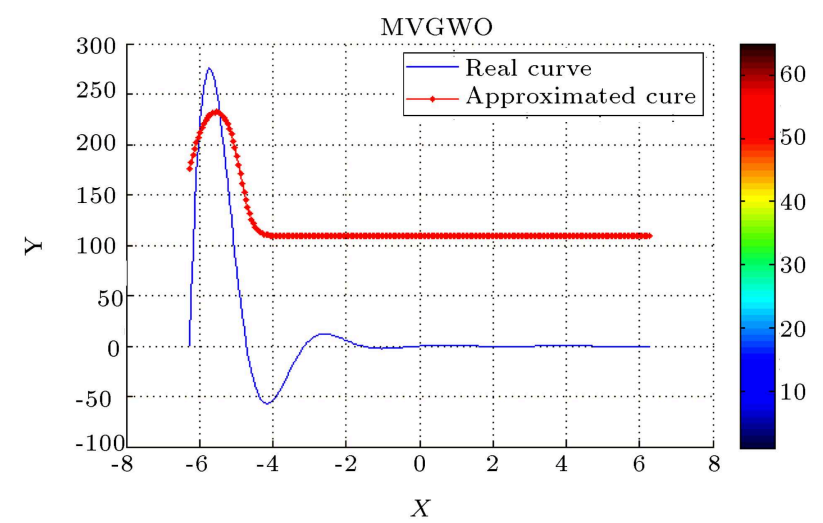

Figure 26. Sine graph of MVGWO.

were compared with several recent nature-inspired algorithms. The results showed that the modified variant proved to be producing effective solutions of sine dataset and cantilever beam design functions as compared to other meta-heuristics.

Table 11. Best optimal solutions of the cantilever beam design function by different meta-heuristics.

\begin{tabular}{lcccccc}
\hline Algorithms & $\boldsymbol{l}$ & $\boldsymbol{m}$ & $\boldsymbol{n}$ & $\boldsymbol{o}$ & $\boldsymbol{p}$ & Min (x) \\
\hline CONLIN [17] & 6.0100 & 5.3000 & 4.4900 & 3.4900 & 2.1500 & NC \\
CS [17] & 6.0089 & 5.3049 & 4.5023 & 3.5077 & 2.1504 & 1.33999 \\
MMA [17] & 6.0100 & 5.3000 & 4.4900 & 3.4900 & 2.1500 & 1.3400 \\
GCA-II [18] & 6.0100 & 5.3000 & 4.4900 & 3.4900 & 2.1500 & 1.3400 \\
GCA-I [18] & 6.0100 & 5.3000 & 4.4900 & 3.4900 & 2.1500 & 1.3400 \\
SOS [19] & 6.01878 & 5.30344 & 4.49587 & 3.49896 & 2.15564 & 1.33996 \\
MVGWO & $\mathbf{6 . 0 1 5 5 4}$ & $\mathbf{5 . 3 0 2 5 6}$ & $\mathbf{4 . 4 9 3 8 6}$ & $\mathbf{3 . 4 9 7 9 7}$ & $\mathbf{2 . 1 5 8 9 6}$ & $\mathbf{1 . 3 3 9 6 6}$ \\
\hline
\end{tabular}




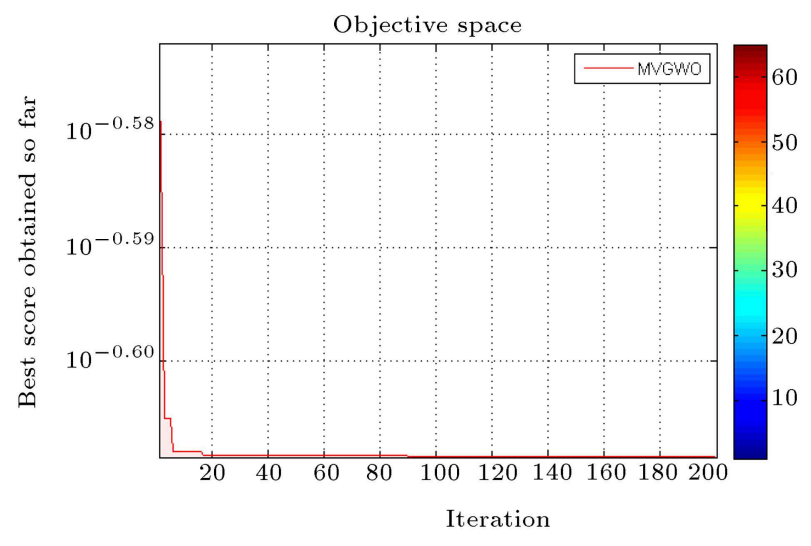

Figure 27. Convergence graph of MVGWO.

\section{References}

1. Holland, J.H. "Genetic algorithms", Scientific American, 267(1), pp. 66-72 (1992).

2. Kennedy, J. and Eberhart, R. "Particle swarm optimization", in Proceedings of the IEEE International Conference on Neural Networks, 4(1), pp. 1942-1948 (1995).

3. Price, K. and Storn, R. "Differential evolution", $D r$. Dobb's Journal, 22(2), pp. 18-20 (1997).

4. Price, K.V., Storn, R.M., and Lampinen, J.A., Differential Evolution: A Practical Approach to Global Optimization, Book, Springer, New York, NY, USA (2005).

5. Wolpert, D.H. and Macready, W.G. "No free lunch theorems for optimization", IEEE Transactions on Evolutionary Computation, 1(1), pp. 67-82 (1997).

6. Karaboga, D. and Basturk, B. "A powerful and efficient algorithm for numerical function optimization: artificial bee colony (ABC) algorithm", Journal of Global Optimization, 39(3), pp. 459-471 (2007).

7. Yang, X.S. and Deb, S. "Cuckoo Search via Levy flights", In Proceedings of the World Congress on Nature \& Biologically Inspired Computing (NaBIC '09), IEEE, Coimbatore, India, pp. 210-214 (2009).

8. Rashedi, E., Nezamabadi-Pour, H., and Saryazdi, S. "GSA: a gravitational search algorithm", Information Sciences, 179(13), pp. 2232-2248 (2009).

9. Yang, X.S. "Firefly algorithm, levy flights and global optimization", In Research and Development in Intelligent Systems XXVI, Springer, London, UK, pp. 209218 (2010).

10. Rajabioun, R. "Cuckoo optimization algorithm", $A p$ plied Soft Computing, 11(8), pp. 5508-5518 (2011).

11. Mirjalili S. and Lewis, A. "Adaptive gbest-guided gravitational search algorithm", Neural Computing and Applications, 25(7), pp. 1569-1584 (2014).

12. Mirjalili, S., Mirjalili, S.M., and Lewis, A. "Grey wolf optimizer", Advances in Engineering Software, 69(2), pp. $46-61$ (2014).
13. Mirjalili, S. "The ant lion optimizer", Advances in Engineering Software, 83(1), pp. 80-98 (2015).

14. Mirjalili, S., Mirjalili, S.M., and Hatamlou, A. "Multiverse optimizer: a nature-inspired algorithm for global optimization", Neural Computing and Applications, 27(2) (2015).

15. Eusuff, M.M. and Lansey, K.E. "Optimization of water distribution network design using the shuffled frog leaping algorithm", Journal of Water Resources Planning and Management, 129(3), pp. 210-225 (2003).

16. Das, S., Biswas, A., Gupta, S.D., and Abraham, A. "Bacterial foraging optimization algorithm: Theoretical foundations, analysis, and applications", In Foundations of Computational Intelligence, Global Optimization, 3(1), pp. 23-55 (2009).

17. Raj, S. and Bhattacharyya, B. "Reactive power planning by opposition-based grey wolf optimization method", International Transactions on Electrical Energy Systems, 28(6) (2018). https://doi.org/10.1002/etep.2551.

18. Singh, N. and Singh, S.B. "One half global best position particle swarm optimization algorithm", International Journal of Scientific \& Engineering Research, 2(8), pp. 1-10 (2011).

19. Singh, N., Singh, S., and Singh, S.B. "Half mean particle swarm optimization algorithm", International Journal of Scientific \& Engineering Research, 3(8), pp. 1-9 (2012).

20. Singh, N. and Singh, S.B. "Personal best position particle swarm optimization", Journal of Applied Computer Science \& Mathematics, 12(6), pp. 69-76 (2012).

21. Singh, N., Singh, S., and Singh, S.B. "HPSO: A new version of particle swarm optimization algorithm", Journal of Artificial Intelligence, 3(3), pp. 123-134 (2012).

22. Singh, N., Singh, S., and Singh, S.B. "A new hybrid MGBPSO-GSA variant for improving function optimization solution in search space", Evolutionary Bioinformatics, 13(1), pp. 1-13 (2017).

23. Singh, N., and Singh, S.B. "A modified mean grey wolf optimization approach for benchmark and biomedical problems", Evolutionary Bioinformatics, 13(1), pp. 128 (2017).

24. Singh, N., and Singh, S.B. "Hybrid algorithm of particle swarm optimization and grey wolf optimizer for improving convergence performance", Journal of Applied Mathematics, Article ID 2030489, 2017, pp. 1-15 (2017). https://doi.org/10.1155/2017/2030489

25. Singh, N. and Singh, S.B. "A novel hybrid GWOSCA approach for optimization problems", Engineering Science and Technology, an International Journal, Elsevier, 20(6) (2017).

https://doi.org/10.1016/j.jestch.2017.11.001

26. Singh, N. and Hachimi, H. "A new hybrid whale optimizer algorithm with mean strategy of grey wolf optimizer for global optimization", Mathematical and Computational Applications, 23(14), pp. 1-32 (2018). 
27. Simon, D. "Biogeography-based optimization", Evolutionary Computation, IEEE Transactions on, 12(6), pp. 702-713 (2008).

28. Yang, X.S. "A new metaheuristic bat-inspired algorithm", Nature Inspired Cooperative Strategies for Optimization (NICSO 2010), 284, pp. 65-74 (2010).

29. Yang, X.S. "Flower pollination algorithm for global optimization", Unconventional Computation and Natural Computation, 7445, pp. 240-249 (2012).

30. Mirjalili, S. "How effective is the grey wolf optimizer in training multi-layer perceptrons", Applied Intelligence, Springer, 43(1), pp. 150-161 (2015).

31. Singh, N. and Singh, S.B. "A modified mean grey wolf optimization approach for benchmark and biomedical problems", Evolutionary Bioinformatics, 13(1), pp.128 (2017).

32. Mittal, N., Singh, U., and Sohi, B.S. "Modified grey wolf optimizer for global engineering optimization", Applied Computational Intelligence and Soft Computing, Article id 7950348, 2016, pp. 1-16 (2016). https://doi.org/10.1155/2016/7950348

33. Emary, E., Zawbaa, H.M., Grosan, C., and Hassenian, A.E. "Feature subset selection approach by gray-wolf optimization", In Afro-European Conference for Industrial Advancement of Advances in Intelligent Systems and Computing, Springer, 334 (2015).

34. Kamboj, V.K., Bath, S.K., and Dhillon, J.S. "Solution of non-convex economic load dispatch problem using grey wolf optimizer", Neural Computing and Applications, 27(5), pp. 1301-1316 (2015).

35. Komaki, G.M. and Kayvanfar, V. "Grey wolf optimizer algorithm for the two-stage assembly flow shop scheduling problem with release time", Journal of Computational Science, 8(2), pp. 109-120 (2015).

36. Gholizadeh, S. "Optimal design of double layer grids considering nonlinear behaviour by sequential grey wolf algorithm", Journal of Optimization in Civil Engineering, 5(4), pp. 511-523 (2015).

37. Yusof, Y. and Mustaffa, Z. "Time series forecasting of energy commodity using grey wolf optimizer", In Proceedings of the International Multi Conference of Engineers and Computer Scientists (IMECS '15), 1(1), Hong Kong (2015).

38. Shankar, K. and Eswaran, P. "A secure visual secret share (VSS) creation scheme in visual cryptography using elliptic curve cryptography with optimization technique", Australian Journal of Basic \& Applied Science, 9(36), pp. 150-163 (2015).

39. El-Fergany, A.A. and Hasanien, H.M. "Single and multi-objective optimal power flow using grey wolf optimizer and differential evolution algorithms", Electric Power Components and Systems, 43(13), pp. 15481559 (2015).

40. Kamboj, V.K. "A novel hybrid PSOGWO approach for unit commitment problem", Neural Computing and Applications (2015).
41. Emary, E., Zawbaa, H.M., and Hassanien, A.E. "Binary grey wolf optimization approaches for feature selection", Neurocomputing, 172(2), pp. 371-381 (2016).

42. Pan, T.S., Dao, T.K., Nguyen, T.T., and Chu, S.C. "A communication strategy for paralleling grey wolf optimizer", Advances in Intelligent Systems and Computing, 388, pp. 253-262 (2015).

43. Jayapriya, J. and Arock, M. "A parallel GWO technique for aligning multiple molecular sequences", In Proceedings of the International Conference on Advances in Computing, Communications and Informatics (ICACCI '15), IEEE, Kochi, India, pp. 210-215 (2015).

44. Zhu, A., Xu, C., Li, Z., Wu, J., and Liu, Z. "Hybridizing grey wolf optimization with differential evolution for global optimization and test scheduling for 3D stacked SoC", Journal of Systems Engineering and Electronics, 26(2), pp. 317-328 (2015).

45. Li, L., Sun, L., Guo, J., Qi, J., Xu, B., and Li, S. "Modified discrete grey wolf optimizer algorithm for multilevel image thresholding", Computational Intelligence and Neuroscience, Article id 3295769, 2017, pp. 1-16 (2017). https://doi.org/10.1155/2017/3295769

46. Liu, H., Hua, G., Yin, H., and Xu, Y. "An intelligent grey wolf optimizer algorithm for distributed compressed sensing", Computational Intelligence Neuroscience, Article id 1723191, 2018, pp. 1-10 (2018). https://doi.org/10.1155/2018/1723191

47. Mirjalili, S., Gandomi, A.H., Mirjalili, S.Z., Saremi, S., Faris, H., and Mirjalili, S.M. "Salp swarm algorithm: A bio-inspired optimizer for engineering design problems", Advances in Engineering Software, 114, pp. 163-191 (2017).

48. Raj, S. and Bhattacharyya, B. "Optimal placement of TCSC and SVC for reactive power planning using whale optimization algorithm", Swarm and Evolutionary Computation, 40, pp. 131-143 (2017).

49. Saremi, S., Mirjalili, S.Z., and Mirjalili, S.M. "Evolutionary population dynamics and grey wolf optimizer", Neural Computing and Applications, 26(5), pp. 12571263 (2015).

50. Mahdad, B. and Srairi, K. "Blackout risk prevention in a smart grid based flexible optimal strategy using grey wolf-pattern search algorithms", Energy Conversion and Management, 98, pp. 411-429 (2015).

51. Lu, Y., Zhou, Y., and Wu, X. "A hybrid lightning search algorithm-simplex method for global optimization", Discrete Dynamics in Nature and Society, Article id 8342694, pp. 1-23 (2017).

52. Chickermane, H. and Gea, H.C. "Structural optimization using a new local approximation method", International Journal for Numerical Methods in Engineering, 39(5), pp. 829-846 (1996).

53. Cheng, M.Y. and Prayogo, D. "Symbiotic organisms search: a new metaheuristic optimization algorithm", Computers \& Structures, 139, pp. 98-112 (2014). 


\section{Biography}

Narinder Singh is a Researcher at the Department of Mathematics, Punjabi University, Patiala, Punjab, India. Dr. Singh obtained his PhD in Mathematics from the Department of Mathematics, Punjabi University, Patiala. He has reviewed several articles for the Hindawi and Scientia Iranica Journals and is a reviewer of seven reputable International Journals. Recently, he has been organizing the special session in "SOFA2018: 8th International Workshop on Soft Computing Applications, September 13-15, 2018, Arad, Romania" and has been invited for a speaker talk concerning "Advanced Focus on Clinical Research and the Future of Biomarkers, September 17-18, 2018 at Toronto, Canada". He has received an invitation to present his paper in the conference going to be held in Zordan in July 2018. He is a member of several association and professional bodies. His primary area of interest lies in nature-inspired optimization techniques. He has published more than 25 research papers in various international journals/conferences. He has also received Gold Medal at M. Phil level and 36 AIR in NET Exam. 